\title{
Riemannian Polyhedra and Liouville-Type Theorems for Harmonic Maps
}

\begin{abstract}
This paper is a study of harmonic maps from Riemannian polyhedra to locally non-positively curved geodesic spaces in the sense of Alexandrov. We prove Liouville-type theorems for subharmonic functions and harmonic maps under two different assumptions on the source space. First we prove the analogue of the Schoen-Yau Theorem on a complete pseudomanifolds with non-negative Ricci curvature. Then we study 2-parabolic admissible Riemannian polyhedra and prove some vanishing results on them.
\end{abstract}

Keywords: Harmonic maps, Riemannian polyhedra, pseudomanifolds, Liouville-type theorem, non-negative Ricci

MSC: 53C23, 31E05, 53C43

DOI 10.2478/agms-2014-0012

Received May 6, 2014; accepted October 14, 2014

\section{Introduction}

Harmonic maps between singular spaces have received considerable attention since the early 1990s. Existence of energy minimizing locally Lipschitz maps from Riemannian manifolds into Bruhat-Tits buildings and Corlette's version of Margulis's super-rigidity theorem were proved in [17]. In [34] Korevaar and Schoen constructed harmonic maps from domains in Riemannian manifolds into Hadamard spaces as a boundary value problem. See [31, 32, 35]. The book [13] by Eells and Fuglede contains a description of the application of the methods of [34] to the study of maps between polyhedra, see also [9, 11, 12].

Our first objective in this paper is to prove Liouville-type theorems for harmonic maps. We prove the analogue of the Schoen-Yau Theorem on complete (smooth) pseudomanifolds with non-negative Ricci curvature. To this end, we generalize some Liouville-type theorems for subharmonic functions from [49].

The classical Liouville theorem for functions on manifolds states that on a complete Riemannian manifold with non-negative Ricci curvature, any harmonic function bounded from one side must be a constant. In [49], Yau proves that there is no non-constant, smooth, non-negative, $L^{p}, p>1$, subharmonic function on a complete Riemannian manifold. He also proves that every continuous subharmonic function defined on a complete Riemannian manifold whose local Lipschitz constant is bounded by $L^{1}$-function is also harmonic. Furthermore if the $L^{1}$-function belongs to $L^{2}$ as well, and the manifold has non-negative Ricci curvature, then the subharmonic function is constant. In the smooth setting, there are two types of assumptions that have been studied on the Liouville property of harmonic maps. One is the finiteness of the energy and the other is the smallness of the image. For example, Schoen and Yau in [45], proved that any non-constant harmonic map from a complete non-compact manifold of non-negative Ricci curvature to a manifold of non-positive sectional curvature has infinite energy. Hildebrandt-Jost-Widman [26] (see also [24, 25]) proved a Liouvilletype theorem for harmonic maps into regular geodesic balls in a complete $C^{3}$-Riemannian manifold from a

^Corresponding Author: Zahra Sinaei: Courant Institute of Mathematical Sciences, New York University, E-mail: sinaei@cims.nyu.edu 
simple or compact $C^{1}$-Riemannian manifold. For more references for Liouville-type theorems for harmonic maps and functions in both smooth and singular setting see the introduction in [39].

A connected locally finite $n$-dimensional simplicial polyhedron $X$ is called admissible, if $X$ is dimensionally $n$-homogeneous and $X$ is locally $(n-1)$-chainable. It is called circuit if instead it is $(n-1)$-chainable and every $(n-1)$-simplex is the face of at most two $n$-simplex and pseudomanifold if it is admissible circuit. A polyhedron $X$ becomes a Riemannian polyhedron when endowed with a Riemannian structure $g$, defined by giving on each maximal simplex $s$ of $X$ a bounded measurable Riemannian metric $g$ equivalent to a Euclidean metric on $s$.

There exist slightly various notions of boundedness of Ricci curvature from below on general metric spaces. See for example $[1,3,14,37,38,41,42,46]$ and the references therein. In what follows by $\operatorname{Ric}_{N, \mu_{g}} \geq K$ we mean that $\left(X, g, \mu_{g}\right)$ satisfies the either $\operatorname{MCP}(K, N)$ or $\operatorname{CD}(K, N)$ unless otherwise specified. As these definitions are somewhat technical we refer the reader to Section 3 for a precise statement.

The definition of harmonic maps from admissible Riemannian polyhedra to metric spaces is similar to the one in the smooth setting. However due to lack of smoothness some care is needed in defining the notions of energy density, the energy functional and energy minimizing maps. Precise definitions and related results can be found in Subsection 2.4.

We can state now the main results which we obtain in this direction.

Theorem 1.1. Suppose $\left(X, g, \mu_{g}\right)$ is a complete, admissible Riemannian polyhedron and $f \in W_{\mathrm{loc}}^{1,2}(X) \cap L^{2}(X)$ is a non-negative, weakly subharmonic function. Then $f$ is constant.

Theorem 1.2. Let $\left(X, g, \mu_{g}\right)$ be a complete, smooth n-pseudomanifold. Suppose $X$ satisfies $\operatorname{CD}(0, n)$. Let $f$ be a continuous, weakly subharmonic function belonging to $W_{\text {loc }}^{1,2}(X)$ such that both $\|\nabla f\|_{L^{1}}$ and $\|\nabla f\|_{L^{2}}$ are finite. Then $f$ is a constant function.

Here by a smooth pseudomanifold we mean a simplexwise smooth pseudomanifold which is smooth outside its singular set. That situation arises for instance when the space is a projective algebraic variety. The difficulty in extending existing results lies in the lack of a differentiable structure on admissible polyhedron in general, and the loss of completeness outside the singular set even in the case of smooth pseudomanifolds. Moreover the classical notion of Laplace operator doesn't exist in the non-smooth setting. To circumvent this latter problem and following the work [15], we define the Laplacian of a subharmonic function as a measure for which the Green formula holds, see Theorem 4.1. Furthermore we prove a Gaffney's Stokes theorem in this setting, see Theorem 4.3.

The following two theorems are corollaries of Theorems 1.1 and 1.2 and are the generalizations of ShoenYau's theorem to the non-smooth setting.

Theorem 1.3. Let $\left(X, g, \mu_{g}\right)$ be a complete, smooth n-pseudomanifold. Suppose $X$ has non-negative $n$-Ricci curvature. Let $Y$ be a Riemannian manifold of non-positive curvature, and $u:(X, g) \rightarrow(Y, h)$ a continuous harmonic map belonging to $W_{l o c}^{1,2}(X, Y)$. If $u$ has finite energy and $e(u)$ is locally bounded, then it is a constant map.

Theorem 1.4. Let $\left(X, g, \mu_{g}\right)$ be a complete, smooth n-pseudomanifold. Suppose $X$ has non-negative $n$-Ricci curvature $\mathrm{CD}(0, n)$. Let $Y$ be a simply connected, complete geodesic space of non-positive curvature and $u$ : $(X, g) \rightarrow Y$ a continuous harmonic map with finite energy, belonging to $W_{\text {loc }}^{1,2}(X, Y)$. If $\int_{M} \sqrt{e(u)} d \mu_{g}<\infty$, then $u$ is a constant map.

Our second objective in this paper is the study of 2-parabolic admissible polyhedra. We say a connected domain $\Omega$ in an admissible Riemannian polyhedron is 2-parabolic if, for every compact set in $\Omega$, its relative capacity with respect to $\Omega$ is zero. Our main theorem is

Theorem 1.5. Let $X$ be 2-parabolic pseudomanifold. Let $f$ in $W_{\text {loc }}^{1,2}(X)$ be a continuous, weakly subharmonic function such that $\|\nabla f\|_{L^{1}}$ and $\|\nabla f\|_{L^{2}}$ are finite. Then $f$ is constant. 
Just as in the case of complete pseudomanifolds

Theorem 1.6. Let $\left(X, g, \mu_{g}\right)$ be a 2-parabolic pseudomanifold with $g$ simplexwise smooth. Let $Y$ be a simply connected complete geodesic space of non-positive curvature and $u:(X, g) \rightarrow Y$ a continuous harmonic map with finite energy belonging to $W_{\text {loc }}^{1,2}(X, Y)$. If we have $\int_{X} \sqrt{e(u)} d \mu_{g}<\infty$, then $u$ is a constant map.

Also we will obtain

Theorem 1.7. Let $\left(X, g, \mu_{g}\right)$ be a 2-parabolic admissible Riemannian polyhedron with simplexwise smooth metric $g$. Let $Y$ be a complete geodesic space of non-positive curvature and $u:(X, g) \rightarrow Y$ a continuous harmonic map belonging to $W_{\text {loc }}^{1,2}(X, Y)$, with bounded image. Then $u$ is a constant map.

In order to prove Theorem 1.5, we need to generalize some of the results in [27] to our setting. This is done in Section 5 in Propositions 5.3, 5.5 and 5.4. The proof of Propositions 5.3 and 5.5 follow a similar pattern as their equivalents for Riemannian manifolds. They are based on the fact that admissible Riemannian polyhedra admit exhaustions by regular domains and the validity of comparison principle on admissible Riemannian polyhedra. The main new ingredient in the proof of Theorem 1.5 is Proposition 5.4.

The rest of this paper is organized as follows. In Section 2, we give a complete background on Riemannian polyhedra and analysis on them. Most definitions and results have been taken directly from [13]. In Subsection 2.2, we compare the $L^{2}$ based Sobolev space on admissible Riemannian polyhedra as in [13] with the one in [10], and show that they are equivalent. As we could not find references in the literature we provide a rather detailed explanation of this fact. In Section 3, we discuss the definition of two notions of Ricci curvature, the curvature dimension condition $\operatorname{CD}(K, N)$ and the measure contraction property $\operatorname{MCP}(K, N)$ on metric measure spaces. We show that both notions are applicable to Riemannian polyhedra. In Proposition 3.6 we show that any non-compact complete $n$-dimensional Riemannian polyhedron of non-negative Ricci curvature has infinite volume. Subsection 4.1 is devoted to Theorems 1.1, 4.3, 1.2 and Subsection 4.2 to Theorems 1.3, and 1.4. In Section 5 we show that as in the smooth case the "approximation by unity" property holds on admissible 2-parabolic polyhedra, see Lemma 5.1. Moreover, we prove that removing the singular set of a 2-parabolic pseudomanifold yields a 2-parabolic manifold (Lemma 5.2). The rest of this Section is the detailed proof of Theorem 1.5 and its corollaries.

\section{Preliminaries}

\subsection{Riemannian polyhedra}

In this subsection we recall the definitions and results about Riemannian polyhedra which will be used in the rest of the manuscript. We refer the reader to the book [13] for more complete discussion on the subject.

\section{Simplicial complex}

A countable locally finite simplicial complex $K$ consists of a countable set $\{v\}$ of elements called vertices and a set $\{s\}$ of finite non-void subsets of vertices called simplexes such that

i. any set consisting of exactly one vertex is a simplex.

ii. any non-void subset of a simplex is a simplex.

iii. every vertex belongs to only finitely many simplexes (the local finiteness condition).

To the simplicial complex $K$, we associate a metric space $|K|$ defined as follows. The space $|K|$ of $K$ is the set of all formal finite linear combinations $\alpha=\sum_{v \in K} \alpha(v) v$ of vertices of $K$ such that $0 \leq \alpha(v) \leq 1, \sum_{v \in K} \alpha(v)=1$ and $\{v: \alpha(v)>0\}$ is a simplex of $K .|K|$ is made into a metric space with barycentric distance $\rho(\alpha, \beta)$ between 
two points $\alpha=\sum \alpha(v) v$ and $\beta=\sum \beta(v) v$ of $|K|$ given by the finite sum

$$
\rho(\alpha, \beta)=\left(\sum_{v \in K}(\alpha(v)-\beta(v))^{2}\right)^{\frac{1}{2}} .
$$

With this metric $|K|$ is locally compact and separable. The metric $\rho$ is not intrinsic. We denote by $\bar{\rho}(\alpha, \beta)$ the length metric associated to $\rho$ by the standard procedure [7].

Lemma 2.1 ([13]). Let $K$ be a countable, locally finite simplicial complex of finite dimension $n$ and $V$ a Euclidean space of dimension $2 n+1$. There exists an affine Lipschitz homeomorphism i of $|K|$ onto a closed subset of $V$.

We shall use the term polyhedron to mean a connected locally compact separable Hausdorff space $X$ for which there exists a simplicial complex $K$ and a homeomorphism $\theta$ of $|K|$ onto $X$. Any such pair $T=(K, \theta)$ is called a triangulation of $X$.

A Lipschitz polyhedron is a metric space $X$ which is the image of the metric space $|K|$ of some complex $K$ under a Lipschitz homeomorphism $\theta:|K| \rightarrow X$. The pair $(K, \theta)$ is then called a Lipscitz triangulation (or briefly a triangulation) of the Lipschitz polyhedron $X$. A null set in a Lipschitz polyhedron $X$ is understood a set $Z \subset X$ such that $Z$ meets every maximal simplex $s$ (relative to some, and hence any triangulation $T=(K, \theta)$ of $X$ ) in a set whose preimage under $\theta$ has $p$-dimensional Lebesgue measure $0, p=\operatorname{dim} s$.

From Lemma 2.1 follows that every Lipschitz polyhedron $\left(X, d_{X}\right)$ can be mapped Lipschitz homeomorphically and (simplexwise) affinely onto a closed subset of a Euclidean space.

\section{Riemannian Structure on a polyhedron}

The class of domains that we consider for our harmonic maps are Riemannian polyhedra. A Riemannian polyhedron is a Lipschitz polyhedron $(X, d)$ such that for some triangulation $T=(K, \theta)$, there exist a measurable Riemannian metric $g^{s}=g_{i j} d x^{i} d x^{j}$ on each maximal simplex $s$ of $i(|K|)(i$ as in Lemma 2.1), which satisfies

$$
\Lambda^{-2}\|\xi\|^{2} \leq g_{i j}(x) \xi^{i} \xi^{j} \leq \Lambda^{2}\|\xi\|^{2}
$$

almost everywhere in standard coordinate in the simplex $s$. Here the constant $\Lambda$ is independent of a given simplex. The distance $d_{X}^{g}$ on $X$ is an intrinsic distance with respect to the metric $g$, meaning that $d^{g}=d_{X}^{g}$ is the infimal length of admissible path joining $x$ to $y$. Actually $\left(X, d^{g}\right)$ is a length space. The detailed definition is somewhat subtle and we refer to [13], for a careful discussion of Riemannian polyhedra.

A Riemannian metric $g$ on a polyhedron $X$ is said to be continuous, if relative to some (hence any) triangulation, $g_{s}$ is continuous up to the boundary on each maximal simplex $s$ and for any two maximal simplexes $s$ and $s^{\prime}$ sharing a face $t, g_{s}$ and $g_{s^{\prime}}$ induce the same Riemannian metric on $t$. There is a similar notion of a Lipschitz continuous Riemannian metric.

A Riemannian polyhedron has a well defined volume element given simplexwise by

$$
d \mu_{g}=\sqrt{\operatorname{det}\left(g_{i j}(x)\right)} d x_{1} d x_{2} \ldots d x_{n},
$$

this measure coincide with Hausdorff measure.

\section{Further definitions}

A polyhedron $X$ will be called admissible if in some (hence in any) triangulation

i. $X$ is dimensionally homogeneous, i.e. all maximal simplexes have the same dimension $n(=\operatorname{dim} X)$, or equivalently every simplex is a face of some $n$-simplex.

ii. $X$ is locally (n-1)-chainable, i.e. for every connected open set $U \subset X$, the open set $U \backslash X^{n-2}$ is connected. 
The boundary $\partial X$ of a polyhedron $X$ is the union of all non-maximal simplexes contained in only one maximal simplex. In this work we always assume that $(X, g)$ satisfies $\partial X=\emptyset$.

By an $n$-circuit we mean a polyhedron $X$ of homogeneous dimension $n$ such that in some (and hence any) triangulation,

i. every $(n-1)$-simplex is a face of at most two $n$-simplexes (exactly two if $\partial X=\emptyset$ ).

ii. $X$ is $(n-1)$-chainable, i.e. $X \backslash X^{n-2}$ is connected, or equivalently any two $n$-simplexes can be joined by a chain of contiguous $(n-1)$ - and $n$-simplexes.

Let $S=S(X)$ denote the singular set of an $n$-circuit $X$, i.e. the complement of the set of all points of $X$ having a neighborhood which is a topological $n$-manifold (possibly with boundary). $S$ is a closed triangulable subspace of $X$ of codimension $\geq 2$ and $X \backslash S$ is a topological $n$-manifold which is dense in $X$. An admissible circuit is called a pseudomanifold. We call a pseudomanifold $\left(X, g, d_{X}\right)$ a Lipschitz pseudomanifold, if $g$ is Lipschitz continuous. If $g$ is simplexwise smooth such that $\left(X \backslash S,\left.g\right|_{X \backslash S}\right)$ has the structure of a smooth Riemannian manifold, we call $\left(X, g, d_{X}\right)$ a smooth pseudomanifold. ${ }^{1}$

\subsection{The Sobolev space $W^{1,2}(X)$}

Let $\left(X, g, d_{X}\right)$ denote an admissible Riemannian polyhedron of dimension $n$. We denote by $\operatorname{Lip}^{1,2}(X)$ the linear space of all Lipschitz continuous functions $u:\left(X, d_{X}\right) \rightarrow \mathbb{R}$ for which the Sobolev $(1,2)$-norm $\|u\|$ defined by

$$
\|u\|_{1,2}^{2}=\int_{X}\left(u^{2}+|\nabla u|^{2}\right) d \mu_{g}=\sum_{s \in S^{(n)}(X)} \int_{S}\left(u^{2}+|\nabla u|^{2}\right) d \mu_{g}
$$

is finite, $S^{(n)}(X)$ denoting the collection of all $n$-simplexes $s$ of $X$ and $|\nabla u|$ the Riemannian norm of the Riemannian gradient on each $s$ (The Riemannian gradient is defined a.e. in $X$ or a.e. in each $s \in S^{n}(X)$ by Rademacher's theorem for Lipschitz functions on Euclidean domains).

The Lebesgue space $L^{2}(X)$ is likewise defined with respect to the volume measure. The Sobolev space $W^{1,2}(X)$ is defined as the completion of $\operatorname{Lip}^{1,2}(X)$ with respect to the Sobolev norm $\|\cdot\|_{1,2}$. We use the notations $\operatorname{Lip}_{c}(X), W_{0}^{1,2}(X)$, and $W_{\text {loc }}^{1,2}(X)$ for the linear space of functions in $\operatorname{Lip}(X)$ with compact support, the closure of $\operatorname{Lip}_{c}(X)$ in $W^{1,2}(X)$ and all $u \in L_{\text {loc }}^{2}(X)$ such that $u \in W^{1,2}(U)$ for all relatively compact subdomains $U$ in $X$.

\section{Sobolev spaces on metric spaces}

Here we recall a few basic notions on analysis on metric spaces. For the sake of completeness, we compare the $L^{2}$ based Sobolev space on admissible Riemannian polyhedra as in [13] with the one in [10] and show that they are equivalent. We use [10] as our main reference. See also [2, 21-23, 44] and [6] for further references.

Let $(Y, d, \mu)$ be a metric measure space, $\mu$ a Borel regular measure. Assume also the measure of balls of finite and positive radius are finite and positive. Fix a set $A \subset Y$. Let $f$ be a function on $A$ with values in the extended real numbers.

Definition 2.2. An upper gradient for $f$ is an extended real valued Borel function $g: A \rightarrow[0, \infty]$ such that for all points $y_{1}, y_{2} \in A$ and all continuous rectifiable curves $c:[0, l] \rightarrow A$ parameterized by arc length $s$ with $c(0)=y_{1}, c(l)=y_{2}$, we have

$$
\left|f\left(y_{2}\right)-f\left(y_{1}\right)\right| \leq \int_{0}^{l} g(c(s)) d s .
$$

1 In many texts the term pseudomanifold is used for what we called a circuit. 
Note that in above definition the left-hand side is interpreted as $\infty$, if either $f\left(y_{1}\right)= \pm \infty$ or $f\left(y_{2}\right)= \pm \infty$. If on the other hand, the right-hand side is finite then it follows that $f(c(s))$ is a continuous function of $s$. For a Lipschitz function $f$ we define the lower pointwise Lipschitz constant of $f$ at $x$ as

$$
\operatorname{lip} f(x)=\liminf _{r \rightarrow 0} \sup _{y \in B(x, r)} \frac{|f(y)-f(x)|}{r} .
$$

$\operatorname{lip} f$ is Borel, finite and bounded by the Lipschitz constant. Also $\operatorname{lip} f$ is an upper gradient for $f$. Similarly for Lipschitz function $f$, the upper pointwise Lipschitz constant $f$, $\operatorname{Lip} f$, is the Borel function

$$
\operatorname{Lip} f(x)=\limsup _{r \rightarrow 0} \sup _{y \in B(x, r)} \frac{|f(y)-f(x)|}{r} .
$$

For any Lipschitz function $f$, we have $\operatorname{lip} f(x) \leq \operatorname{Lip} f$. In the special case $Y=\mathbb{R}^{n}$, if $x$ is a point of differentiability of $f$, we observe that $\operatorname{lip} f(x)=\operatorname{Lip} f(x)=|\nabla f(x)|$. We now define the Sobolev space $H^{1, p}$ for $1 \leq p<\infty$.

Definition 2.3. Whenever $f \in L^{p}(Y)$, let

$$
\|f\|_{1, p}=\|f\|_{L^{p}}+\inf _{g_{i}} \liminf _{i \rightarrow \infty}\left\|g_{i}\right\|_{L^{p}}
$$

where the infimum is taken over all sequence $\left\{g_{i}\right\}$, for which there exists a sequence $f_{i} \stackrel{L^{p}}{\rightarrow} f$, such that $g_{i}$ is an upper gradient for $f_{i}$ for all $i$.

For $p \geq 1$, the Sobolev space $H^{1, p}$, is the subspace of $L^{p}$ consisting of functions $f$ for which $\|f\|_{1, p}<\infty$ equipped with the norm $\|\cdot\|_{1, p}$. The space $H^{1, p}$ is complete.

We define now the notions of generalized upper and minimal upper gradients. This will allow us to give a nice interpretation of the $H^{1, p}$ norm of Sobolev functions.

Definition 2.4. We say

i. A function $g \in L^{p}$ is a generalized upper gradient for $f \in L^{p}$, if there exist sequences $f_{i} \stackrel{L^{p}}{\rightarrow} f, g_{i} \stackrel{L^{p}}{\rightarrow} g$, such that $g_{i}$ is an upper gradient for $f_{i}$ for all $i$.

ii. For fixed $p$, a minimal generalized upper gradient for $f$ is a generalized upper gradient $g_{f}$ such that $\|f\|_{1, p}=\|f\|_{L^{p}}+\left\|g_{f}\right\|_{L^{p}}$.

The following theorem ensures the existence of minimal generalized upper gradient for any Sobolev function.

Theorem 2.5 ([10]). For all $1<p<\infty$ and $f \in H^{1, p}$, there exists a minimal generalized upper gradient $g_{f}$, which is unique up to modification on subsets of measure zero.

We will discuss two important properties of metric spaces called the ball doubling property and the Poincaré inequality for functions on them. These are essential assumptions to get a richer theory on metric spaces.

Definition 2.6. Let $(Y, d, \mu)$ be a metric measure space. The measure $\mu$ is said to be locally doubling if for all $r^{\prime}$, there exists $\kappa=\kappa\left(r^{\prime}\right)$ such that for all $y \in Y$ and $0<r<r^{\prime}$,

$$
0<\mu\left(B_{r}(y)\right) \leq 2^{\kappa} \mu\left(B_{r / 2}(y)\right) .
$$

Definition 2.7. Let $q \geq 1$. We say that $Y$ supports a weak Poincaré inequality of type $(q, p)$ if for all $r^{\prime}>0$, there exist constants $1 \leq \lambda<\infty$ and $C=C\left(p, r^{\prime}\right)>0$ such that for all $r \leq r^{\prime}$ and all upper gradients $g$ of $f$,

$$
\left(\int_{B_{r}(x)}\left|f-f_{x, r}\right|^{q} d \mu\right)^{1 / q} \leq C r\left(\int_{\lambda B_{r}(x)}|g|^{p} d \mu\right)^{1 / p}
$$

where $f_{x, r}:=\int_{B_{r}(x)} f d \mu$. If $\lambda=1$, then we say that $X$ supports a strong $(q, p)$-Poincaré inequality. 
For every admissible Riemannian polyhedron $\left(X, g, \mu_{g}\right), \mu_{g}$ is locally doubling. Moreover $X$ supports a weak $(2,2)$-Poincaré inequality and by Hölder's inequality $(1,2)$-Poincaré inequality (see Corollary 4.1 and Theorem (5.1) in [13]). In the sequel, the words "Poincaré inequality" refer to (2, 2)-Poincaré inequality.

By Theorem 4.24 in [10] for any metric space which satisfies (2.2) and (2.3), for some $1 \leq p<\infty$ and $q=1$, the subspace of locally Lipschitz functions is dense in $H^{1, p}$. Furthermore, on a locally complete metric space with the mentioned properties, for some $1<p<\infty$ and for any $f$ locally lipschitz, we have $g_{f}=\operatorname{Lip} f, \mu$ almost everywhere (see [10] Theorem 6.1). Therefore, on a Riemannian polyhedron $\left(X, g, \mu_{g}\right)$, for any $f \in H^{1,2}$, $g_{f}(y)=|\nabla f(y)|$ for a.e. $y$ and it follows that $H^{1,2}$ is equivalent to $W^{1,2}$.

In the following, we always consider $X=\left(X, g, \mu_{g}\right)$ to be an admissible Riemannian polyhedron. Some of the concepts below are defined on metric spaces in general but for simplicity we present them only on Riemannian polyhedron and for $p=2$. For more information on metric spaces we refer the reader to [6].

\subsection{Potential theory background}

In this subsection we recall some of the definitions in potential theory. First we define different notions of capacities (see [6]).

\section{Sobolev and variational capacities}

The Sobolev capacity of a set $E \subset X$ is the number

$$
C(E)=\inf \|u\|_{W^{1,2}(X)}^{2}
$$

where the infimum is taken over all $u \in W^{1,2}(X)$ such that $u \geq 1$ on $E$.

Let $E \subset \Omega \subset X$ and $\Omega$ bounded. The variational capacity is defined as

$$
\operatorname{cap}(E, \Omega)=\inf _{u} \int_{\Omega}|\nabla u|^{2} d \mu_{g}
$$

where the infimum is taken over all $u \in W_{0}^{1,2}(\Omega)$ such that $u \geq 1$ on $E$. In this definitions the infimum can be taken only over $u \leq 1$ such that it is equal 1 on a neighborhood of $E$. Also we write $\operatorname{cap}(E)=\operatorname{cap}(E, X)$.

A set $U \subset X$ is quasi open if there are open sets $\omega$ of arbitrarily small capacity such that $U \backslash \omega$ is open relative to $X \backslash \omega$. A map $\phi: U \rightarrow Y$ from a quasiopen set $U$ to a topological space $Y$ with a countable base of open sets is quasicontinuous if there are open sets $\omega$ of arbitrarily small capacity such that $\left.\phi\right|_{U \backslash \omega}$ is continuous. Clearly this amounts to $\phi^{-1}(V)$ being quasiopen for every open subset $V$ of $Y$.

\section{Weakly harmonic and weakly sub/super harmonic functions}

A function $u \in W_{l o c}^{1,2}(X)$ is said to be weakly harmonic if

$$
\int_{X}\langle\nabla u, \nabla \rho\rangle d \mu_{g}=0 \quad \text { for every } \rho \in \operatorname{Lip}_{c}(X) .
$$

It is said to be weakly subharmonic, respectively weakly superharmonic, if

$$
\int_{X}\langle\nabla u, \nabla \rho\rangle d \mu_{g} \leq 0, \text { resp. } \geq 0 \quad \text { for every } \rho \in \operatorname{Lip}_{c}(X) .
$$


A function $u \in W^{1,2}(X)$ is weakly harmonic if and only if $u$ minimizes the energy $E(v)$ among all functions $v \in W^{1,2}(X)$ such that $v-u \in W_{0}^{1,2}(X)$ (see [13]). In the following we discuss on the existence of minimizer under specific assumption on the Riemannian polyhedra.

Theorem 2.8 ([13]). Suppose the following Poincaré inequality holds

$$
\int_{X}|u|^{2} d \mu_{g} \leq c \int_{X}|\nabla u|^{2} d \mu_{g} \quad \text { for all } u \in W_{0}^{1,2}(X)
$$

with $c$ depending only on the admissible Riemannian polyhedron X. For any $f \in W^{1,2}(X)$ the class of competing maps

$$
W_{f}^{1,2}(X)=\left\{v \in W^{1,2}(X): v-f \in W_{0}^{1,2}(X)\right\}
$$

contains a unique weakly harmonic function $u$. That function is the unique minimizer of $E(u)=E_{0}$, where

$$
E_{0}:=\inf \left\{E(v): v \in W^{1,2}(X), v-f \in \operatorname{Lip}_{c}(X)\right\}=\min \left\{E(v): v \in W_{f}^{1,2}(X)\right\} .
$$

As a corollary of the above theorem we have

Corollary 2.9. Assume that the domain $\Omega \subset X$ is bounded and such that the Sobolev capacity $C(X \backslash \Omega)>0$. For any $f \in W^{1,2}(\Omega)$, the class of functions

$$
W_{f}^{1,2}(\Omega)=\left\{v \in W^{1,2}(\Omega): v-f \in W_{0}^{1,2}(\Omega)\right\}
$$

has a unique solution $u$ of the equation $E(u)=E_{\Omega}$, where

$$
E_{\Omega}:=\inf \left\{E(v): v \in W^{1,2}(\Omega), v-f \in W_{0}^{1,2}(\Omega)\right\} .
$$

Proof. Since $X$ satisfies the Poincaré inequality and using Theorem 5.54 in [6], $\Omega$ satisfies the inequality (2.5). By the above theorem, there is a unique minimizer which is weakly harmonic.

After correction on a null set every weakly harmonic function on $X$ is Hölder continuous. A continuous weakly harmonic function is called harmonic.

Remark 2.10. From the discussion above one can see in the definition of variational capacity that there is a harmonic function $u$ which takes the minimum in (2.4). This function is not necessarily continuous on the boundary of $\Omega \backslash E$.

\section{Polar sets}

A set $S \subset X$ is said to be a polar set for the capacity if for every pair of relatively compact open sets $U_{1} \Subset U_{2} \subset X$ such that $d\left(U_{1}, X \backslash U_{2}\right)>0$ we have

$$
\operatorname{cap}\left(S \cap \bar{U}_{1}, U_{2}\right)=0 .
$$

According to Theorem 9.52 in [6] (see also section 3 in [19]), $S$ is a polar set if and only if every point of $X$ has an open neighborhood $U$ on which there is a superharmonic function which equals $+\infty$ at every point of $S \cap U$. An equivalent formulation is to say that $C(S)=0$.

Lemma 2.11. A closed set $S \subset X$ is a polar set if and only if for every neighborhood $U$ of $S$ and every $\epsilon>0$, there exists a function $\operatorname{Lip}(X)$ such that

i. the support of $\varphi$ is contained in $X \backslash S$.

ii. $0 \leq \varphi \leq 1$. 
iii. $\varphi \equiv 1$ on $X \backslash U$.

iv. $\int_{X}|\nabla \varphi|^{2}<\epsilon$.

Proof. The proof is based on the definition of polar set and followed by a similar argument as the case of Riemannian manifolds. See Proposition 3.1 in [47] for the proof of the equivalence on Riemannian manifolds.

\section{The Dirichlet space $L_{0}^{1,2}(X)$}

In this part we introduce the Dirichlet space $L_{0}^{1,2}(X)$ on an admissible Riemannian polyhedron $X$ (see [13] for the definition of Dirichlet spaces). The Dirichlet space $L_{0}^{1,2}(X)$ determines a Brelot harmonic structure on $X$. Using this fact we can show, $X$ has a symmetric Green function which gives us information on the singularities of $X$.

Proposition 2.12. [13] Suppose that, for every compact set $K \subset X$,

$$
\left(\int_{K}|u| d \mu_{g}\right)^{2} \leq c(K) E(u) \quad \text { for all } x \in \operatorname{Lip}_{c}(X),
$$

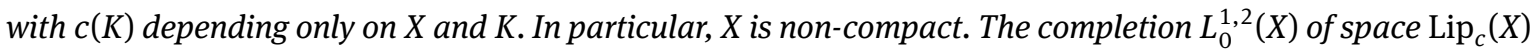
within $L_{\text {loc }}^{1}(X)$ with respect to the norm $E(u)^{1 / 2}$ is then a regular Dirichlet space of strongly local type. $L_{0}^{1,2}(X)$ is a subset of $W_{\text {loc }}^{1,2}(X)$.

Note that $W_{0}^{1,2}(X) \subset L_{0}^{1,2}(X) \subset W_{\text {loc }}^{1,2}(X)$. Let

$$
\Delta: L_{0}^{1,2}(X) \supset D(\Delta) \rightarrow L^{2}(X)
$$

denote the generator induced from $\left(L_{0}^{1,2}(X), E\right)$, which is a densely defined non-positive definite self-adjoint operator satisfying $E(u, v)=(\Delta u, v)_{L^{2}}$. Here $D(\Delta)$ denotes the domain of operator $\Delta$. We have

Theorem 2.13 ([13]). Let $\left(X, g, \mu_{g}\right)$ be an admissible Riemannian polyhedra such that the inequality (2.7) holds. Then $X$ has a unique symmetric Green kernel

$$
G: X \times X \rightarrow(0, \infty]
$$

which is finite and Hölder continuous off the diagonal $X \times X$.

For local questions, condition (2.7) is not required (it is automatically satisfied with $X$ replaced by the open star of a point $a$ of $X$ relative to a sufficiently fine triangulation and in view of inequality (2.3)). As a consequence of Theorem 2.13, we have

Proposition 2.14. The $(n-2)$-skeleton $X^{(n-2)}$ of an admissible Riemannian n-polyhedron is a polar set.

We should note that being polar is independent of the Riemannian structure on the polyhedron.

Remark 2.15. Every closed polar subset $F$ of $X$ is removable for Sobolev functions, $W^{1,2}(X \backslash F)=W^{1,2}(X)$. A larger class of removable sets in this sense is that of all (closed) sets of $(n-1)$-dimensional Hausdorff measure zero (see Proposition 7.7 in [13]).

\subsection{Harmonic maps on Riemannian polyhedra}

The energy of a map from a Riemannian domain to an arbitrary metric space was defined and investigated by Korevaar and Schoen [34]. Here we give an introduction to the concept of energy of maps, energy minimizing 
maps and harmonic maps on a Riemannian polyherdron. In the case that the target $Y$ is a Riemannian $C^{1}$ manifold, the energy of the map is given by the usual expression (similarly when the target is a Riemannian polyhedron with continuous Riemannian metric).

Let $(X, g)$ be an admissible $n$-dimensional Riemannian polyhedron with simplexwise smooth Riemannian metric. We do not require that $g$ is continuous across lower dimensional simplexes. Let $Y$ be an arbitrary metric space. Denote by $L_{\text {loc }}^{2}(X, Y)$ the space of all $\mu_{g}$-measurable maps $\varphi: X \rightarrow Y$ having separable essential range ${ }^{2}$ and for which $d_{Y}(\varphi(\cdot), q) \in L_{\text {loc }}^{2}\left(X, \mu_{g}\right)$ for some point $q$ (and therefore by the triangle inequality for any $q \in Y)$. For $\varphi, \psi \in L_{\mathrm{loc}}^{2}(X, Y)$ define their distance

$$
D(\varphi, \psi)=\left(\int_{X} d_{Y}^{2}(\varphi(x), \psi(x)) d \mu_{g}(x)\right)^{1 / 2} .
$$

The approximate energy density of a map $\varphi \in L_{\text {loc }}^{2}(X, Y)$ is defined for $\varepsilon>0$ by

$$
e_{\varepsilon}(\varphi)(x)=\int_{B(x, \varepsilon)} \frac{d_{Y}^{2}\left(\varphi(x), \varphi\left(x^{\prime}\right)\right)}{\varepsilon^{n+2}} d \mu_{g}\left(x^{\prime}\right) .
$$

The function $e_{\varepsilon}(\varphi)$ is of class $L_{\text {loc }}^{1}\left(X, \mu_{g}\right)$ (see [34]).

Definition 2.16. The energy $E(\varphi)$ of a map $\varphi$ of class $L_{\text {loc }}^{2}(X, Y)$ is defined as

$$
E(\varphi)=\sup _{f \in C_{c}(X,[0,1])}\left(\limsup _{\varepsilon \rightarrow 0} \int_{X} f e_{\varepsilon}(\varphi) d \mu_{g}\right) .
$$

We say that $\varphi$ is locally of finite energy and write $\varphi \in W_{\text {loc }}^{1,2}(X, Y)$, if $E\left(\left.\varphi\right|_{U}\right)<\infty$ for every relatively compact domain $U \subset X$. For example every Lipschitz continuous map $\varphi: X \rightarrow Y$ is in $W_{\text {loc }}^{1,2}(X, Y)$. Now we give a necessary and sufficient condition for a map $\varphi$ to be in $W_{\text {loc }}^{1,2}(X, Y)$.

Lemma 2.17. Let $(X, g)$ be an admissible $n$-dimensional Riemannian polyhedron with simplexwise smooth Riemannian metric and $\left(Y, d_{Y}\right)$ a metric space. A map $\varphi \in L_{\text {loc }}^{2}(X, Y)$ is locally of finite energy if and only if there is a function $e(\varphi) \in L_{\text {loc }}^{1}(X)$ such that $e_{\varepsilon}(\varphi) \rightarrow e(\varphi)$ as $\varepsilon \rightarrow 0$, in the sense of weak convergence of measures

$$
\lim _{\varepsilon \rightarrow 0} \int_{X} f e_{\varepsilon}(\varphi) d \mu_{g}=\int_{X} f e(\varphi) d \mu_{g} \quad f \in C_{c}(X) .
$$

\section{Energy of maps into Riemannian manifolds}

Let the domain be an arbitrary admissible Riemannian polyhedron $(X, g)$ ( $g$ is only measurable with local elliptic bounds, unless otherwise specified) and the target is a Riemannian $C^{1}$-manifold $(N, h)$ without boundary, $X$ of dimension $n$ and $Y$ of dimension $m$.

A chart $\eta$ of $N, \eta: V \rightarrow \mathbb{R}^{m}$ is bi-Lipschitz if the components $h_{\alpha \beta}$ of $\left.h\right|_{V}$ have elliptic bounds

$$
\Lambda_{V}^{-2} \sum_{\alpha=1}^{m}\left(\eta^{\alpha}\right)^{2} \leq h_{\alpha \beta} \eta^{\alpha} \eta^{\beta} \leq \Lambda_{V}^{2} \sum_{\alpha=1}^{m}\left(\eta^{\alpha}\right)^{2} .
$$

Relative to a given countable atlas on a Riemannian $C^{1}$-manifold $(N, h)$, a map $\varphi:(X, g) \rightarrow(N, h)$ is of class $W_{\text {loc }}^{1,2}(X, N)$, or locally of finite energy, if

2 The essential range of a map $\varphi$ is a closed set of points $q \in Y$ such that for any neighborhood $V$ of $q, \varphi^{-1}(V)$ has positive measure. 
i. $\varphi$ is a quasicontinuous (after correction on a set of measure zero).

ii. Its components $\varphi_{1}, \ldots, \varphi_{m}$ in charts $\eta: V \rightarrow \mathbb{R}^{m}$ are of class $W^{1,2}(U)$ for every quasiopen set $U \subset$ $\varphi^{-1}(V)$ of compact closure in $X$.

iii. The energy density $e(\varphi)$ of $\varphi$, defined a.e. in each of the quasiopen sets $\varphi^{-1}(V)$ covering $X$ by

$$
e(\varphi)=\left(h_{\alpha \beta} \circ \varphi\right) g\left(\nabla \varphi^{\alpha}, \nabla \varphi^{\beta}\right)
$$

is locally integrable over $\left(X, \mu_{g}\right)$.

The energy of $\varphi \in W_{\text {loc }}^{1,2}(X, N)$ is defined by $E(\varphi)=\int_{X} e(\varphi) d \mu_{g}$.

\section{Energy minimizing maps}

We suppose that $(X, g), n$-dimensional admissible Riemannian polyhedra with $g$ simplexwise smooth and $Y$ any metric space. A map $\varphi \in W_{\text {loc }}^{1,2}(X, Y)$ is said to be locally energy minimizing if $X$ can be covered by relatively compact domains $U \subset X$ for which $E\left(\left.\phi\right|_{U}\right) \leq E\left(\left.\psi\right|_{U}\right)$ for every map $\psi \in W_{\text {loc }}^{1,2}(X, Y)$ such that $\varphi=\psi$ a.e. in $X \backslash U$.

\section{Harmonic maps}

Consider an admissible Riemannian polyhedron $(X, g)$, of dimension $n$, and a metric space $\left(Y, d_{Y}\right)$,

Definition 2.18. A harmonic map $\varphi: X \rightarrow Y$ is a continuous map of class $\varphi \in W_{\text {loc }}^{1,2}(X, Y)$, which is locally energy minimizing in the sense that $X$ can be covered by relatively compact subdomains $U$, for each of which there is an open set $V \supset \varphi(U)$ in $Y$ such that

$$
E\left(\left.\varphi\right|_{U}\right) \leq E\left(\left.\psi\right|_{U}\right)
$$

for every continuous map $\psi \in W_{\text {loc }}^{1,2}(X, Y)$ with $\psi(U) \subset V$ and $\varphi=\psi$ in $X \backslash U$.

Every continuous, locally energy minimizing map $\varphi: X \rightarrow Y$ is harmonic. Also if $Y$ is a simply connected complete Riemannian polyhedron of non-positive curvature, then a harmonic map $\varphi: X \rightarrow Y$ is the same as a continuous locally energy minimizing map.

We proceed now to characterize harmonic maps are continuous, weakly harmonic maps. We consider here $(X, g)$ to be an arbitrary admissible Riemannian polyhedron and $g$ just bounded measurable with local elliptic bounds, $X$ of dimension $n$, and $(N, h)$ a smooth Riemannian manifold without boundary, and the dimension of $N$ is $m$. We denote by $\Gamma_{\alpha \beta}^{k}$ the Christoffel symbols on $N$.

Definition 2.19. A weakly harmonic map $\varphi: X \rightarrow N$ is a quasicontinuous map of class $W_{\text {loc }}^{1,2}(X, N)$ with the following property: for any chart $\eta: V \rightarrow \mathbb{R}^{n}$ on $N$ and any quasiopen set $U \subset \varphi^{-1}(V)$ of compact closure in $X$, the equation

$$
\int_{U}\left\langle\nabla \lambda, \nabla \varphi^{k}\right\rangle d \mu_{g}=\int_{U} \lambda \cdot\left(\Gamma_{\alpha \beta}^{k} \circ \varphi\right)\left\langle\nabla \varphi^{\alpha}, \nabla \varphi^{\beta}\right\rangle d \mu_{g}
$$

holds for every $k=1, \ldots, m$ and every bounded function $\lambda \in W_{0}^{1,2}(U)$.

According to [13], a continuous map $\varphi \in W_{\text {loc }}^{1,2}(X, N)$ is harmonic (Definition 2.18) if and only if it is weakly harmonic (Definition 2.19). 


\section{Ricci Curvature on Riemannian Polyhedra}

In the past few years, several notions of boundedness of Ricci curvature from below on general metric spaces have appeared. Sturm [46] and Lott-Villani [41] independently introduced the so called curvature-dimension condition on a metric measure space denoted by $\operatorname{CD}(K, N)$. The curvature dimension condition implies the generalized Brunn-Minkowski inequality (hence the Bishop-Gromov comparison and Bonnet-Myer's theorem) and a Poincaré inequality (see [40, 41, 46]). Meanwhile, Sturm and Ohta introduced a measure contraction property denoted as $\operatorname{MCP}(K, N)$ in Ohta's work. The condition $\operatorname{MCP}(K, N)$ also implies the Bishop-Gromov comparison, Bonnet-Myer's theorem and a Poincaré inequality (see [42, 46]). Note that all of these generalized notions of Ricci curvature bounded below are equivalent to the classical one on smooth Riemannian manifolds. Then after the reduced curvature dimension condition $\operatorname{CD}^{\star}(K, N)$ has been introduce by Bacher and Sturm in [5] to overcome local-to-global property and it is equivalent to local $\operatorname{CD}(K, N)$ condition. More recently the notion of Riemannian curvature dimension condition $\operatorname{RCD}(K, \infty)$ has been introduced in [1] and it is equivalent to the $\mathrm{CD}(K, \infty)$ on infinitesimally Hilbertian metric measure spaces. The finite dimensional version of this notion $\operatorname{RCD}(K, N)$ has been introduced in [14] and independently in [3] and it is equivalent to $\mathrm{CD}^{\star}(K, N)$ on infinitesimally Hilbertian metric measure spaces.

Here we define both $\operatorname{CD}(K, N), \operatorname{MCP}(K, N)$ and show that on a Riemannian polyhedron we can use both of them. In the following definitions, we always assume that $(X, d)$ is a separable length space, $P(X)$ is the set of all Borel probability measures $\mu$ satisfying $\int_{X} d_{X}(x, y)^{2} d \mu(y)<\infty$ for some $x \in X . P_{2}(X)$ is the set $P(X)$ equipped with the $L^{2}$-Wasserstein distance $W_{2}$ defined as

$$
W_{2}\left(\mu_{0}, \mu_{1}\right)^{2}=\inf _{\pi} \int_{X \times X} d\left(x_{0}, x_{1}\right)^{2} d \pi\left(x_{0}, x_{1}\right)
$$

for $\mu_{0}, \mu_{1}$ in $P_{2}(X)$ and $\pi$ in $P(X \times X)$ ranges between all transference plan between $\mu_{0}$ and $\mu_{1}$. A transference plan is defined as

$$
p_{0 \star}(\pi)=\mu_{0} \quad p_{1 \star}(\pi)=\mu_{1}
$$

where $p_{0}, p_{1}: X \times X \rightarrow X$ are projection to the first and second factors respectively.

\section{Curvature Dimension Condition}

We now define the notion of spaces satisfying $\operatorname{CD}(K, N)$ condition following [41]. Suppose $(X, d)$ is a compact length space. Let $U:[0, \infty) \rightarrow \mathbb{R}$ be a continuous convex function with $U(0)=0$. We define the non-negative function

$$
p(r)=r U_{+}^{\prime}(r)-U(r)
$$

with $p(0)=0$. Given a reference probability measure $v \in P_{2}(X)$, define the function $U_{v}: P_{2}(X) \rightarrow \mathbb{R} \cup\{\infty\}$ by

$$
U_{v}(\mu)=\int_{X} U(\rho(x)) d v(x)+U^{\prime}(\infty) \mu_{s}(X)
$$

where

$$
\mu=\rho v+\mu_{s}
$$

is the Lebesgue decomposition of $\mu$ with respect to $v$ into an absolutely continuous part $\rho v$ and a singular part $\mu_{s}$, and

$$
U^{\prime}(\infty)=\lim _{r \rightarrow \infty} \frac{U(r)}{r}
$$


If $N \in[1, \infty)$ then we define $\mathcal{D} \mathcal{C}_{\mathcal{N}}$ to be the set of such functions $U$ so that

$$
\psi(\lambda)=\lambda^{N} U\left(\lambda^{-N}\right)
$$

is convex on $(0, \infty)$. We further define $\mathcal{D} \mathcal{C}_{\infty}$ to be the set of such functions $U$ so that the function

$$
\psi(\lambda)=e^{\lambda} U\left(e^{-\lambda}\right)
$$

is convex on $(-\infty, \infty)$. A relevant example of an element in $\mathcal{D} \mathcal{C}_{\mathcal{N}}$ is given by

$$
H_{N, v}= \begin{cases}N r\left(1-r^{-1 / N}\right) & \text { if } 1<N<\infty, \\ r \log r & \text { if } N=\infty\end{cases}
$$

\section{Definition 3.1.}

i. Given $N \in[1, \infty]$, we say that a compact measured length space $(X, d, v)$ has non-negative $N$-Ricci curvature if for all $\mu_{0}, \mu_{1} \in P_{2}(X)$ with $\operatorname{supp}\left(\mu_{0}\right) \subset \operatorname{supp}(v)$ and $\operatorname{supp}\left(\mu_{1}\right) \subset \operatorname{supp}(v)$, there is some Wasserstein geodesic $\left\{\mu_{t}\right\}_{t \in[0,1]}$ from $\mu_{0}$ to $\mu_{1}$ so that for all $U \in \mathcal{D} \mathcal{C}_{\mathcal{N}}$ and all $t \in[0,1]$,

$$
U_{v}\left(\mu_{t}\right) \leq t U_{v}\left(\mu_{1}\right)+(1-t) U_{v}\left(\mu_{0}\right) .
$$

ii. Given $K \in \mathbb{R}$, we say that $(X, d, v)$ has $\infty$-Ricci curvature bounded below by $K$ if for all $\mu_{0}, \mu_{1} \in P_{2}(X)$ with $\operatorname{supp}\left(\mu_{0}\right) \subset \operatorname{supp}(v)$ and $\operatorname{supp}\left(\mu_{1}\right) \subset \operatorname{supp}(v)$, there is some Wasserstein geodesic $\left\{\mu_{t}\right\}_{t \in[0,1]}$ from $\mu_{0}$ to $\mu_{1}$ so that for all $U \in \mathcal{D} \mathcal{C}_{\infty}$ and all $t \in[0,1]$,

$$
U_{v}\left(\mu_{t}\right) \leq t U_{v}\left(\mu_{1}\right)+(1-t) U_{v}\left(\mu_{0}\right)-\frac{1}{2} \lambda(U) t(1-t) W_{2}\left(\mu_{0}, \mu_{1}\right)^{2}
$$

where $\lambda: \mathcal{D C} \mathcal{C}_{\infty} \rightarrow \mathbb{R} \cup\{-\infty\}$ is defined as

$$
\lambda(U)=\inf _{r>0} K \frac{p(r)}{r}= \begin{cases}K \lim _{r \rightarrow 0^{+}} \frac{p(r)}{r} & \text { if } K>0, \\ 0 & \text { if } K=0, \\ K \lim _{r \rightarrow \infty} \frac{p(r)}{r} & \text { if } K<0 .\end{cases}
$$

Note that inequalities (3.2) and (3.3) are only assumed to hold along some Wasserstein geodesic from $\mu_{0}$ to $\mu_{1}$, and not necessarily along all such geodesics. This is what is called weak displacement convexity.

Proposition 3.2. If a compact measured length space $(X, d, v)$ has non-negative $N$-Ricci curvature for some $N \in[1, \infty)$, then for all $x \in \operatorname{supp}(v)$ and all $0<r_{1} \leq r_{2}$ the following inequality holds

$$
v\left(B_{r_{2}}(x)\right) \leq\left(\frac{r_{2}}{r_{1}}\right)^{N} v\left(B_{r_{1}}(x)\right) .
$$

To generalize the notion of $N$-Ricci curvature to the non-compact case, we always consider a complete pointed locally compact metric measure space $\left(X,{ }^{\star}, v\right)$. Also for $U_{v}$ to be a well-defined functional on $P_{2}(X)$, we impose the restriction $v \in M_{-2(N-1)}$, where $M_{-2(N-1)}$ is the space of all non-negative Radon measures $v$ on $X$ such that

$$
\int_{X}\left(1+d\left({ }^{\star}, x\right)^{2}\right)^{-(N-1)} d v(x)<\infty .
$$

We define $M_{-\infty}$ by the condition $\int_{X} e^{-c d\left(\star^{\star}, x\right)^{2}} d v(x)<\infty$, where $c$ is a fixed positive constant. We should mention that most of the results for compact case (for example the Bishop-Gromov comparison) are valid for the non-compact case. 


\section{Measure Contraction Property}

We define now the notion of measure contraction property $\operatorname{MCP}(K, N)$ following [42]. Let $\left(X, d_{X}\right)$ be a length space and $\mu$ a Borel measure on $X$ such that $0<\mu(B(x, r))<\infty$ for every $x \in X$ and $r>0$, where $B(x, r)$ denotes the open ball with center $x \in X$ and radius $r>0$.

Let $\Gamma$ be the set of minimal geodesics $\gamma:[0,1] \rightarrow X$ and define the evaluation map $e_{t}$ by $e_{t}(\gamma):=\gamma(t)$ for each $t \in[0,1]$. We regard $\Gamma$ as a subset of the set of Lipschitz maps $\operatorname{Lip}([0,1], X)$ with the uniform topology. A dynamical transference plan $\Pi$ is a Borel probability measure on $\Gamma$ and a path $\left\{\mu_{t}\right\}_{t \in[0,1]} \subset P_{2}(X)$ given by $\mu_{t}=\left(e_{t}\right)_{*} \Pi$ is called a displacement interpolation associated to $\Pi$. For the exact definition of dynamical transference plan and displacement interpolation we refer the reader to [41]. For $K \in \mathbb{R}$, we define the function $s_{K}$ on $[0, \infty)($ on $[0, \pi / \sqrt{K})$ if $K>0)$ by

$$
s_{K}(t):= \begin{cases}(1 / \sqrt{K}) \sin (\sqrt{K} t) & \text { if } K>0, \\ t & \text { if } K=0, \\ (1 / \sqrt{-K}) \sinh (\sqrt{-K} t) & \text { if } K<0 .\end{cases}
$$

Definition 3.3. For $K, N \in \mathbb{R}$ with $N>1$, or with $K \leq 0$ and $N=1$, a metric measure space $(X, d, \mu)$ is said to satisfy the $(K, N)$-measure contraction property $\operatorname{MCP}(K, N)$, if for every point $x \in X$ and measurable set $A \subset X$ (provided that $A \subset B(x, \pi \sqrt{(N-1) / K})$ if $K>0$ ) with $0<\mu(A)<\infty$, there exists a displacement interpolation $\left\{\mu_{t}\right\}_{t \in[0,1]} \subset P_{2}(X)$ associated to a dynamical transference plan $\Pi=\Pi_{x, A}$ satisfying

i. We have $\mu_{0}=\delta_{X}$ and $\mu_{1}=\left(\left.\mu\right|_{A}\right)^{-}$as measures where we denote by $\left(\left.\mu\right|_{A}\right)^{-}$the normalization of $\left.\mu\right|_{A}$, i.e. $\left(\left.\mu\right|_{A}\right)^{-}:=\left.\mu(A)^{-1} \cdot \mu\right|_{A}$.

ii. For every $t \in[0,1]$,

$$
d \mu \geq\left(e_{t}\right) \star\left(t\left\{\frac{s_{K}(t l(\gamma) / \sqrt{N-1})}{s_{K}(l(\gamma) / \sqrt{N-1})}\right\}^{N-1} \mu(A) d \Pi(\gamma)\right)
$$

holds as measures on $X$, where we set $0 / 0=1$ and by convention, we read

$$
\left\{\frac{s_{K}(t l(\gamma) / \sqrt{N-1})}{s_{K}(l(\gamma) / \sqrt{N-1})}\right\}^{N-1}=1
$$

if $K \leq 0$ and $N=1$.

Here we state two results that we are going to use in the sequel.

Proposition 3.4. Let $(M, g)$ be an $n$-dimensional, complete Riemannian manifold without boundary with $n \geq 2$. Then a metric measure space $\left(M, d_{g}, v_{g}\right)$ satisfies the $\operatorname{MCP}(K, n)$ if and only if $\operatorname{Ric}_{g} \geq K$ holds. Here $d_{g}$ and $v_{g}$ denote the Riemannian distance and Riemannian volume element.

In the following theorem we state Bishop volume comparison theorem for the space satisfying $\operatorname{MCP}(K, N)$.

Proposition 3.5. Let $(X, \mu)$ be a metric space satisfying the $\operatorname{MCP}(K, N)$. Then for any $x \in X$, the function

$$
\mu(B(x, r)) \cdot\left\{\int_{0}^{r} s_{K}\left(\frac{s}{\sqrt{N-1}}\right)^{N-1} d_{s}\right\}^{-1}
$$

is monotone non-increasing in $r \in(0, \infty)\left(r \in\left(0, \pi \sqrt{\frac{N-1}{K}}\right)\right.$ if $\left.K>0\right)$.

In the following we show that we can apply both measure contraction property and curvature dimension condition to a complete Riemannian polyhedra $\left(X, g, \mu_{g}\right)$. By previous section, a Riemannian polyhedron $\left(X, g, \mu_{g}\right)$ with the metric $d_{X}=d_{X}^{g}$ is a length space. Also for any $x, y \in X$ we have

$$
e(x, y) \leq d_{X}^{e}(x, y)
$$


where $e(x, y)$ denotes the Euclidian distance. It is easy then to show that $\mu_{g}$ is in $M_{-2(N-1)}$ and so on a complete Riemannian polyhedron we can use the notion of $\operatorname{CD}(K, N)$. Also $\mu_{g}$ is Borel and by Lemma 4.4 in [13], for any $r$ there exist a constant $c(r)$ such that

$$
c(r)^{-1} \Lambda^{-2 n} r^{n} \leq \mu_{g}(B(x, r)) \leq c(r) \Lambda^{2 n} r^{n}
$$

for all $x \in X$. Therefore $0<\mu_{g}(B(x, r))<\infty$ and the notion of $\operatorname{MCP}(K, N)$ is also applicable for $N \geq n$. By Theorem 2.4.3 in [4], we have the Hausdorff dimension is $n$ and by Corollary 2.7 in [42] $N$ should be greater than $n$.

In the rest of this work by $\operatorname{Ric}_{N, \mu_{g}} \geq K$ can be taken to mean that $\left(X, g, \mu_{g}\right)$ satisfies either $\operatorname{MCP}(K, N)$ or $\operatorname{CD}(K, N)$ unless otherwise specified. In the following Lemma we show that any complete Riemannian polyhedron with non-negative Ricci curvature has infinite volume.

Lemma 3.6. Let $\left(X, g, \mu_{g}\right)$ be a complete, non-compact Riemannian polyhedron of dimension $n$. If $\operatorname{Ric}_{N, \mu_{g}}(X) \geq$ 0 for $N \geq n$, then $X$ has infinite volume.

Proof. First we consider the case $\operatorname{MCP}(0, N)$. By the Bishop comparison theorem, Theorem 3.5, for $x \in X$ and all $0<r_{1} \leq r_{2}$,

$$
\mu_{g}\left(B_{r_{2}}(x)\right) \leq\left(\frac{r_{2}}{r_{1}}\right)^{N} \mu_{g}\left(B_{r_{1}}(x)\right) .
$$

By Proposition 10.1.1 in [43] for every point in $X$, there exist a geodesic ray from that point. Consider a geodesic ray $\gamma(t), 0 \leq t<\infty$, such that $\gamma(0)=x$. We construct the balls $B(\gamma(t), t-1)$ and $B(\gamma(t), t+1)$ centered at $\gamma(t)$ with radius $t-1$ and $t+1$. We have

$$
\frac{\mu_{g}(B(\gamma(0), 1))+\mu_{g}(B(\gamma(t), t-1))}{\mu_{g}(B(\gamma(t), t-1))} \leq \frac{\mu_{g}(B(\gamma(t), t+1))}{\mu_{g}(B(\gamma(t), t-1))} \leq\left(\frac{t+1}{t-1}\right)^{N},
$$

and so

$$
1+\frac{\mu_{g}(B(\gamma(0), 1))}{\mu_{g}(B(\gamma(t), t-1))} \leq\left(\frac{t+1}{t-1}\right)^{N} .
$$

Letting $t \rightarrow \infty$, we get $\mu_{g}(B(\gamma(t), t-1)) \rightarrow \infty$ and therefore $X$ has infinite volume. By Theorem 3.2, and since $X$ is a complete locally compact length space, we can repeat the proof for the case when $X$ satisfies the non-negative $N$-Ricci curvature condition $\operatorname{CD}(0, N)$, for $N \in(1, \infty)$.

Here we recall some remarks that we need through the rest of this paper.

\section{Remark 3.7.}

i. By Remark 5.8 in [46] if $(X, d, \mu)$ satisfy $\operatorname{MCP}(K, N)$ so does any convex set $A \subset X$. When $X$ is a smooth pseudomanifold, for any point $x \in X \backslash S$, there exist a closed totally convex neighborhood $V$ around $x$ (for every point in a Riemannian manifold there is a geodesic ball which is totally convex). Therefore if $X$ satisfies $\operatorname{Ric}_{N, \mu_{g}} \geq K$, so does $X \backslash S$. The same result is valid on metric measure spaces with $\operatorname{CD}(K, N)$ condition, see Theorem 5.53 in [41].

ii. By definition of $\operatorname{CD}^{*}(K, N), \mathrm{CD}^{*}(0, N)$ is equivalent to $\operatorname{CD}(0, N)$.

iii. Since the Sobolov space $W^{1,2}(X)$ on an admissible Riemannian polyhedra $\left(X, g, \mu_{g}\right)$ is a Hilbert space, then $X$ is infinitesimally Hilbertian ${ }^{3}$. Therefore the notion $\operatorname{RCD}(K, \infty)$ is equivalent to $\operatorname{CD}(K, \infty)$ and $\operatorname{RCD}(0, N)$ is equivalent to $\operatorname{CD}(0, N)$.

3 see Definition 4.18 in [15] for the definition of infinitesimally Hilbertian. 


\section{Some Function Theoretic Properties On Complete Riemannain Polyhedra}

\subsection{Liouville-type Theorems for Functions}

The aim of this section is to generalize some of the results in [49] in order to prove some vanishing theorems for harmonic maps on Riemannian polyhedra. In [49], Yau used the Gaffney's Stokes theorem on complete Riemannian manifolds to prove that every smooth subharmonic function with bounded $\|\nabla f\|_{L_{1}}$ is harmonic. He uses this fact to prove that there is no non-constant $L^{p}, p>1$, non-negative subharmonic function on a complete manifold. We generalize this theorem to complete admissible polyhedra for $p=2$ as stated in Theorem 1.1.

Proof of Theorem 1.1. Fix a base point $x_{0} \in X$ and define $\rho: X \rightarrow \mathbb{R}$ as

$$
\rho(x)=\max \left\{0, \min \left\{1,2-\frac{1}{R} d\left(x, x_{0}\right)\right\}\right\}
$$

Observe that $\rho$ is $\frac{1}{R}$-Lipschitz and $\rho=0$ on $X \backslash B\left(x_{0}, 2 R\right)$ and $\rho=1$ on $B\left(x_{0}, R\right)$. Since $f$ is subharmonic,

$$
\begin{aligned}
0 & \geq \int_{X}\left\langle\nabla\left(\rho^{2} f\right), \nabla f\right\rangle d \mu_{g} \\
& =\int_{X}\left\langle\left(\nabla \rho^{2}\right) f+(\nabla f) \rho^{2}, \nabla f\right\rangle d \mu_{g} \\
& =\frac{1}{2} \int_{X}\left\langle\nabla \rho^{2}, \nabla f^{2}\right\rangle d \mu_{g}+\int_{X} \rho^{2}|\nabla f|^{2} d \mu_{g} \\
& =2 \int_{X}\langle\rho \nabla \rho, f \nabla f\rangle d \mu_{g}+\int_{X} \rho^{2}|\nabla f|^{2} d \mu_{g} .
\end{aligned}
$$

From Cauchy-Schwarz we have

$$
\int_{X}\langle\rho \nabla \rho, f \nabla f\rangle d \mu_{g}=\int_{X}\langle f \nabla \rho, \rho \nabla f\rangle d \mu_{g} \geq-\left(\int_{X}|f \nabla \rho|^{2} d \mu_{g}\right)^{\frac{1}{2}}\left(\int_{X}|\rho \nabla f|^{2} d \mu_{g}\right)^{\frac{1}{2}} .
$$

Combining the two previous inequalities, we obtain

$$
\begin{aligned}
0 & \geq 2 \int_{X}\langle\rho \nabla \rho, f \nabla f\rangle d \mu_{g}+\int_{X} \rho^{2}|\nabla f|^{2} d \mu_{g} \\
& \geq \int_{B_{2 R} \backslash B_{R}}|\rho \nabla f|^{2} d \mu_{g}-2\left(\int_{B_{2 R} \backslash B_{R}}|f \nabla \rho|^{2} d \mu_{g}\right)^{\frac{1}{2}}\left(\int_{B_{2 R} \backslash B_{R}}|\rho \nabla f|^{2} d \mu_{g}\right)^{\frac{1}{2}}+\int_{B_{R}}|\nabla f|^{2} d \mu_{g} .
\end{aligned}
$$

The last line is a polynomial, $P(\psi)=\psi^{2}-2 b \psi+c$, where $\psi$ is

$$
\left(\int_{B_{2 R} \backslash B_{R}}|\rho \nabla f|^{2} d \mu_{g}\right)^{\frac{1}{2}}
$$

and it has non-positive value only if $b^{2} \geq c$, which means that

$$
\int_{B_{R}}|\nabla f|^{2} d \mu_{g} \leq \int_{B_{2 R} \backslash B_{R}} f^{2}|\nabla \rho|^{2} \leq \frac{c^{2}}{R^{2}} \int_{B_{2 R}} f^{2} d \mu_{g}
$$


and so

$$
\int_{B_{R}}|\nabla f|^{2} d \mu_{g} \leq \frac{c^{2}}{R^{2}} \int_{X} f^{2} d \mu_{g} .
$$

Sending $R$ to infinity and using the fact that $f$ has finite $L^{2}$-norm, we conclude that

$$
\int_{X}|\nabla f|^{2} d \mu_{g}=0 .
$$

Since $X$ is admissible, $f$ is constant on $X$; first we prove that $f$ is constant on each maximal $n$-simplex $S$ and then using the $n$-1-chainability of $X$, we prove this in the star of any vertex $p$ of $X$ and then by connectedness on $X$.

In the following theorem, we show that the Laplacian of a weakly subharmonic function $f \in W_{\mathrm{loc}}^{1,2}(X)$ on a pseudomanifold in the distributional sense is a locally finite Borel measure. This gives us a verifying of Green's formula on these spaces. We then use this theorem, to prove that a continuous weakly subharmonic function with $\|\nabla f\|_{L^{1}}<\infty$ on a complete normal circuit is harmonic.

Theorem 4.1. Let $\left(X, g, \mu_{g}\right)$ be an n-pseudomanifold. Let $f$ be a weakly subharmonic function in $W_{\mathrm{loc}}^{1,2}(X)$, such that $\|\nabla f\|_{L^{1}}$ is finite. Then there exists a unique locally finite Borel measure $\boldsymbol{m}_{f}$ on $X$ such that

$$
\int_{X} h \boldsymbol{m}_{f}=-\int_{X}\langle\nabla f, \nabla h\rangle d \mu_{g} \quad \text { for all } h \in \operatorname{Lip}_{c}(X) .
$$

Proof. We consider the Lipschitz manifold $M=X \backslash S$ and the chart $\left\{\left(U_{\alpha}, \psi_{\alpha}\right)\right\}$ on $M$. We show that

$$
\Lambda_{\alpha}(h)=-\int_{U_{\alpha}}\left\langle\nabla f, \nabla h \circ \psi_{\alpha}\right\rangle d \mu_{g}
$$

is a linear continuous functional on $D_{\alpha}=\operatorname{Lip}_{c}\left(\psi_{\alpha}\left(U_{\alpha}\right)\right)$ with respect to the topology of uniform convergence on compact sets. The linearity is obvious. We have

$$
\Lambda_{\alpha}(h)=-\int_{U_{\alpha}}\left\langle\nabla f, \nabla h \circ \psi_{\alpha}\right\rangle d \mu_{g} \leq \sup _{x \in U_{\alpha}}|\nabla h(x)| \cdot\|\nabla f\|_{L^{1}\left(U_{\alpha}\right)}
$$

and so $\Lambda_{\alpha}$ is continuous. Since $\operatorname{Lip}_{c}(U)$ is dense in $C_{c}(U)$ for a locally compact domain $U$, see Proposition 1.11 in [6], then $\Lambda_{\alpha}$ is also continuous on $C_{c}\left(\psi_{\alpha}\left(U_{\alpha}\right)\right)$. By assumption $f$ is subharmonic and so $\Lambda_{\alpha}$ is positive. By Riesz representation theorem, $\Lambda_{\alpha}$ is a unique positive Radon measure. It follows that there is a positive Radon measure $m_{\alpha}$ such that

$$
\Lambda_{\alpha}(h)=\int_{U_{\alpha}} h d m_{\alpha} .
$$

Now we consider the partition of unity $\left\{\rho_{\alpha}\right\}$ subordinate to $\left\{U_{\alpha}\right\}$. We put $m=\sum_{\alpha} \rho_{\alpha} \psi_{\star}\left(m_{\alpha}\right)$ and we define $\mathbf{m}_{f}(U)=m(U \backslash S)$ on each Borel set $U$. Obviously $\mathbf{m}_{f}$ is positive and locally finite. The uniqueness comes from the uniqueness of $m_{\alpha}$.

We recall a remark concerning the above theorem.

Remark 4.2. Gigli introduced the notion of Laplacian as a set of locally finite Borel measure (see Definition 4.4 in [15]). There he proved that on infinitesimally Hilbertian spaces this set contains only one element.

In the smooth setting, as a corollary of Gaffney's Stokes theorem, we have that on a complete Riemannian manifold every smooth subharmonic function $f$ with bounded $\|\nabla f\|_{L^{1}}$ is harmonic. We generalize this theorem as follows: 
Theorem 4.3. Let $\left(X, g, \mu_{g}\right)$ be a complete non-compact pseudomanifold. Let $f$ be continuous, weakly subharmonic and belonging to $W_{\text {loc }}^{1,2}(X)$ such that $\|\nabla f\|_{L^{1}}$ is finite. Then $f$ is a harmonic function.

Proof. We put $A_{1}=\|\nabla f\|_{L^{1}}$. We consider a sequence of cut-off functions $\rho_{n}$ for fixed $q \in X$ such that $\rho_{n}$ is $\frac{1}{n}$-Lipschitz and such that $\rho_{n}$ is equal to 1 on $B(q, R)$ and its support is in $B(q, R+n) . f$ is a subharmonic function which satisfies the condition of previous lemma, so there is a unique Borel measure $\mathbf{m}_{f}$ such that

$$
0 \leq \int_{X} \rho_{n} d \mathbf{m}_{f}=-\int_{X}\left\langle\nabla \rho_{n}, \nabla f\right\rangle d \mu_{g} \leq \int_{X}\left|\nabla \rho_{n}\right||\nabla f| d \mu_{g} \leq \frac{1}{n} A_{1}
$$

and

$$
0 \leq \int_{B(q, R)} d \mathbf{m}_{f} \leq \int_{X} \rho_{n} d \mathbf{m}_{f} \leq \frac{1}{n} A_{1} .
$$

Let $h$ be any function in $\operatorname{Lip}_{c}(X)$ with support in $B(q, R)$. We have

$$
0 \leq \int_{X} h d \mathbf{m}_{f} \leq\left(\sup _{X} h\right) \frac{1}{n} A_{1}
$$

and tending $n$ to infinity, we have

$$
\int_{X} h d \mathbf{m}_{f}=-\int_{X}\langle\nabla h, \nabla f\rangle d \mu_{g}=0
$$

and implying that $f$ is harmonic.

Now we prove a generalization of Proposition 2 in [49], see Theorem 1.2 for the exact statement. We give here another proof of the theorem above for smooth pseudomanifolds under the extra assumption that $f$ should have finite energy. Instead of Theorem 4.1, we goal Cheeger's Green formula on compact smooth pseudomanifolds in the proof.

Proof of Theorem 1.2. We put $A_{1}=\|\nabla f\|_{L^{1}}$ and $A_{2}=\|\nabla f\|_{L^{2}}$. We present the proof in several steps.

Step 1. We consider a sequence of cut-off functions $\rho_{n}$ as above such that the support of $\rho_{n}$ is in $B(q, R+n)$ for fixed $q \in X \backslash X^{n-2}$ and some $R$ and $\rho_{n}$ is equal to 1 on $B(q, R)$ and $\rho_{n}$ is $\frac{1}{n}$-Lipschitz.

Step 2. The $(n-2)$-skeleton in $X, X^{n-2}$, is a polar set. We consider shrinking bounded neighborhoods $U_{j}$ of $X^{n-2}$ in $B(q, R+j)$, such that in each $B(q, R+j)$ we have

$$
U_{j} \supset U_{j+1} \supset \ldots \supset \bigcap_{k=1}^{\infty} U_{j} .
$$

By the definition of polar set, for the open domains $U_{j}$ and $U_{j-1}$, we have $\operatorname{cap}\left(X^{n-2} \cap U_{j}, U_{j-1}\right)=0$. This means that for every $j$, there exists a function $\varphi_{j} \in \operatorname{Lip}(X)$ such that $\varphi_{j} \equiv 1$ in a neighborhood of $X^{n-2} \cap U_{j}$ and $\varphi_{j}$ is zero outside $U_{j-1}$ and $\int_{X}\left|\nabla \varphi_{j}\right|^{2}<\frac{1}{j}$. Moreover we have $0 \leq \varphi_{j} \leq 1$.

We put $\eta_{j}=1-\varphi_{j}$. The function $\eta_{j}$ has the property that the closure of its support, $\overline{\operatorname{supp} \eta_{j}}$, is contained in $X \backslash X^{n-2}$ and the set $K_{j}=\overline{\operatorname{supp} \eta_{j}} \cap \overline{B(q, R+j)}$ is compact. Furthermore $K_{j}$ make an exhaustion of $M=X \backslash X^{n-2}$.

Step 3. According to Theorem 2 in [20], for any $j$, there exist a smooth subharmonic function $f_{j}$ on $M$ such that $\sup _{x \in K_{j}}\left|f_{j}(x)-f(x)\right|<\frac{1}{j}$ and $\left|\nabla f_{j}(x)\right| \leq|\nabla f(x)|$ on $K_{j}$.

Step 4. In this step we prove

$$
\int_{M} \Delta f_{j} \cdot \xi_{j} d \mu_{g}=-\int_{M}\left\langle\nabla f_{j}, \nabla \xi_{j}\right\rangle d \mu_{g} .
$$

where $\xi_{j}=\rho_{j} \cdot \eta_{j}$. To prove the above equality, first we recall a Remark from [8]. 
Remark 4.4. Let $(Y, h)$ be a closed $n$-dimensional admissible Riemannian polyhedron, then for $\zeta, \psi \in$ $\operatorname{Dom}(\Delta)$ we have the following Stokes theorem on $Y \backslash Y^{n-2}$ (see Theorem 5.1 in [8]),

$$
\int_{Y \backslash Y^{n-2}} \Delta \zeta \cdot \psi d \mu_{h}=-\int_{Y \backslash Y^{n-2}}\langle\nabla \zeta, \nabla \psi\rangle d \mu_{h} .
$$

Also, every closed smooth pseudomanifold $(Y, h)$ such that $h$ is equivalent to some piecewise flat metric is admissible (in the sense of Cheeger).

Now we construct the closed Riemannian polyhedron $\bar{Y}_{j} \subset X$ as following: Let $Y_{j}$ be an arbitrary Riemannian polyhedron containing $B(q, R+j)$. We consider its double $\tilde{Y}_{j}$ and equip it with a Riemannian metric $\tilde{g}_{j}$, which is the same as Riemannian metric on $Y_{j}$. The Riemannian polyhedron $\bar{Y}_{j}=Y_{j} \cup \tilde{Y}_{j}$ with the metric $\bar{g}_{j}$ is an admissible closed Riemannian pseudomanifold. The metric $g_{j}$ on $Y_{j}$ is equivalent to piecewise flat metric $g^{e}$ (see [13], Chapter 4) and so $\bar{Y}_{j}$ is admissible.

We extend $\rho_{j}$ to $\bar{Y}_{j}$ such that it is zero on the copy of $Y_{j}$ and $f_{j}, \eta_{j}$ such that they are the same functions on the copy of $Y_{j}$. The function $f_{j}$ is in $W_{\text {loc }}^{1,2}\left(\bar{Y}_{j}\right)$ (see Theorem 1.12.3. in [34]).

By applying formula (4.2) on $\bar{Y}_{j}$, for the functions $f_{j}$ and $\xi_{j}$, we obtain

$$
\int_{M_{j}} \Delta f_{j} \cdot \xi_{j} d \mu_{g_{j}}=-\int_{M_{j}}\left\langle\nabla f_{j}, \nabla \xi_{j}\right\rangle d \mu_{g_{j}}
$$

where $M_{j}=\bar{Y}_{j} \backslash \bar{Y}_{j}^{n-2}$. Since $\xi_{j} \in \operatorname{Lip}_{c}(M) \cap Y_{j}$, we can write the above Stokes formula as follows

$$
\int_{M} \Delta f_{j} \cdot \xi_{j} d \mu_{g}=-\int_{M}\left\langle\nabla f_{j}, \nabla \xi_{j}\right\rangle d \mu_{g} .
$$

Step 5. In this step we prove that $f$ is harmonic on $M$. From the fact that $\operatorname{supp}\left(\xi_{j}\right) \subset K_{j}$ we have

$$
\begin{aligned}
\int_{M} \Delta f_{j} \cdot \xi_{j} d \mu_{g} & =-\int_{M}\left\langle\nabla f_{j}, \nabla\left(\rho_{j} \cdot \eta_{j}\right)\right\rangle d \mu_{g} \\
& =-\int_{M} \mid\left\langle\nabla f_{j}, \eta_{j} \cdot\left(\nabla \rho_{j}\right)\right\rangle d \mu_{g}-\int_{M}\left\langle\nabla f_{j}, \rho_{j} \cdot\left(\nabla \eta_{j}\right)\right\rangle d \mu_{g} \\
& \leq \int_{K_{j}}\left|\nabla f_{j}\right|\left|\nabla \rho_{j}\right| d \mu_{g}+\int_{K_{j}}\left|\nabla f_{j}\right|^{2} d \mu_{g} \cdot \int_{K_{j}}\left|\nabla \eta_{j}\right|^{2} d \mu_{g} \\
& \leq \frac{1}{j} \int_{M}|\nabla f| d \mu_{g}+\frac{1}{j} \int_{M}|\nabla f|^{2} d \mu_{g} .
\end{aligned}
$$

So we have

$$
0 \leq \int_{M} \Delta f_{j} \cdot \xi_{j} d \mu_{g} \leq \frac{1}{j}\left(A_{2}+A_{1}\right) .
$$

Let $h$ be any smooth function with compact support in $M \cap B(q, R)$. Then there is a $K_{m}$ such that the support of $h$ is in $B(q, R) \cap K_{m}$. For $j$ large enough we will have $\xi_{j} \equiv 1$ on $K_{m}$ and so we have

$$
0 \leq \int_{B(q, R) \cap K_{m}} \Delta f_{j} d \mu_{g} \leq \frac{1}{j}\left(A_{2}+A_{1}\right) .
$$

considering the formula (4.2) as above, for $j$ large enough we have

$$
0 \leq \int_{M} \Delta h \cdot f_{j} d \mu_{g}=\int_{M} h \cdot \Delta f_{j} d \mu_{g} \leq(\sup h) \cdot \frac{1}{j}\left(A_{2}+A_{1}\right) .
$$

Letting $j$ go to infinity, we got $\int_{M} \Delta h \cdot f d \mu_{g}=0$. By use of Weyl's lemma $f$ is a smooth harmonic function on $M$. 
Step 6. In this step we prove $f$ is locally Lipschitz. Since $f \in W_{\text {loc }}^{1,2}(X)$ and by Theorem 12.2 in [6], $f$ is harmonic on $X$. Then by Corollary 6 in [33] (see also Theorem 3.1 in [30]) $f$ is locally Lipschitz.

Remark 4.5. In [36], the Lipschitz regularity of harmonic functions has been proved on metric measure spaces under the assumptions of Ahlfors regularity of the measure, Poincare inequality and a heat semigroup type curvature condition. In the most recent work of $[29,30,33]$ the Lipschitz regularity of the functions whose Laplacian are either in $L^{p}$ or in $L^{\infty}$ has been studied under more relaxed assumption on the measure. Furthermore the Cheng-Yau gradient estimate has been obtained in [28] for metric measure spaces under $\operatorname{RCD}(K, N)$ curvature dimension condition. See $[16,50]$ for the equivalent results on Alexandrov spaces.

Step 7. Now we show $f$ is constant. Since $M$ has non-negative Ricci curvature, by the Bochner formula $|\nabla f|$ is subharmonic on $M$ and so on $X$ (see Theorem 12.2 in [6]). By Lemma 1.1, $|\nabla f|$ is constant. Since the $L^{2}$-norm of $|\nabla f|$ is finite we have $|\nabla f| \equiv 0$. By Lemma 3.6, $f$ should be constant.

\subsection{Vanishing Results for Harmonic Maps on Complete Smooth PseudoManifolds}

In this subsection we prove Theorems 1.3 and 1.4.

Proof of Theorem 1.3. By Remark 3.7, we know that on the Riemannian manifold $M=X \backslash S$ we have nonnegative Ricci curvature. We show that for $\epsilon>0, \sqrt{e(u)+\epsilon}$ is weakly subharmonic on $X$. As the restriction maps $u=\left.u\right|_{M}:(M, g) \rightarrow Y$ is harmonic, we have a Bochner type formula for harmonic map on $M$ and

$$
\Delta e(u)>|B(u)|^{2}
$$

where $B(u)$ is the second fundamental form of the map $u$. Also by Cauchy-Schwarz we have

$$
|\nabla e(u)|^{2} \leq 2 e(u)|B(u)|^{2}
$$

and so for $\epsilon>0$, on $X \backslash S$

$$
\Delta \sqrt{e(u)+\epsilon} \geq 0 .
$$

See e.g. the calculation in [48] Theorem 1.3.8. Thus $\sqrt{e(u)+\epsilon}$ is subharmonic on $X \backslash S$ and by Theorem 12.2 in [6], subharmonicity on $X$ follows since $S$ is polar and $e(f)$ is locally bounded. Therefore

$$
\int_{X}\langle\nabla \sqrt{e(u)+\epsilon}, \nabla \rho\rangle d \mu_{g} \leq 0 \quad \rho \in \operatorname{Lip}_{c}(X) .
$$

As in the proof of Theorem 1.1,

$$
\int_{B_{R}}|\nabla \sqrt{e(u)+\epsilon}|^{2} d \mu_{g} \leq \frac{1}{R^{2}} \int_{B_{2 R}} e(u)+\epsilon d \mu_{g} .
$$

Note that $\sqrt{e(u)+\epsilon}$ satisfies all the assumptions of the Theorem 1.1, except the finiteness of $L^{2}$-norm which we do not need in this step.

Set $B_{R}^{\prime}=B_{R} \backslash\left\{x \in B_{R}, e(u)(x)=0\right\}$. Then

$$
\int_{B_{R}^{\prime}} \frac{|\nabla(e(u)+\epsilon)|^{2}}{4(e(u)+\epsilon)} d \mu_{g} \leq \frac{1}{R^{2}} \int_{B_{2 R}} e(u)+\epsilon d \mu_{g} .
$$

Letting $\epsilon \rightarrow 0$ gives

$$
\int_{B_{R}^{\prime}} \frac{|\nabla e(u)|^{2}}{4 e(u)} d \mu_{g} \leq \frac{1}{R^{2}} \int_{B_{2 R}} e(u) d \mu_{g}
$$


and letting $R \rightarrow \infty$ and by finiteness of the energy we have

$$
\int_{B_{R}^{\prime}} \frac{|\nabla e(u)|^{2}}{4 e(u)} d \mu_{g} \leq 0
$$

which implies that $e(u)$ is constant. If $e(u)$ is not zero everywhere this means that the volume of $X$ is finite. By Lemma 3.6, this is impossible and so $u$ is constant.

Now we prove Theorem 1.4. By the following lemma, the function $d(u(\cdot), q)$, where $q$ is an arbitrary point in $Y$, is subharmonic under suitable assumption on the curvature of $Y$. We refer the reader to [13] Lemma 10.2, for the proof.

Lemma 4.6. Let $(X, g)$ be an admissible Riemannian polyhedron, $g$ simplexwise smooth. Let $\left(Y, d_{Y}\right)$ be a simply connected complete geodesic space of non-positive curvature, and let $u \in W_{\text {loc }}^{1,2}(X, Y)$ be a locally energy minimizing map. Then $u$ is a locally essentially bounded map and for any $q \in Y$, the function $d(u(\cdot), q)$ of class $W_{\text {loc }}^{1,2}(X, Y)$ is weakly subharmonic and in particular essentially locally bounded.

We have

Proof of Theorem 1.4. According the lemma above the function $v(x)=d\left(u(x), u\left(x_{0}\right)\right)$ for some $x_{0} \in X$, is weakly subharmonic. We know that $|\nabla v|^{2} \leq c e(u)$, where $c$ is a constant. $v$ is a continuous subharmonic function whose gradient is bounded by an $L^{1}$ and $L^{2}$ integrable function. According to Lemma 1.1, $v$ is a constant function and so $u$ is a constant map.

Remark 4.7. Using above argument we have also showed every continuous harmonic map $u:(X, g) \rightarrow Y$ belonging to $W_{\text {loc }}^{1,2}(X, Y)$ with $\int_{M} \sqrt{e(u)} d \mu_{g}<\infty$, where $\left(X, g, \mu_{g}\right)$ is a complete, non-compact $n$-pseudomanifold with non-negative $n$-Ricci curvature $\operatorname{CD}(0, n)$ and $Y$ a simply connected, complete geodesic space of nonpositive curvature, is Lipschitz continuous.

\section{2-Parabolic Riemannian Polyhedra}

In this last section we prove Liouville-type theorems for harmonic maps defined on a Riemannian polyhedra $X$ without any completeness or Ricci curvature bound assumption. We assume instead $X$ to be 2-parabolic. Some of these results extend known results for the case of Riemannian manifolds. As for Riemannian manifolds, we say that a domain $\Omega \subset X$ in an admissible Riemannian polyhedra $X$ is 2-parabolic, if $\operatorname{cap}(D, \Omega)=0$ for every compact set $D$ in $\Omega$, otherwise 2-hyperbolic. A reference on this subject is [19], where the notion is discussed for general metric measure spaces. Our main results in this section are Theorems 1.6 and 1.7. We will first need the following characterization of 2-parabolicity.

Lemma 5.1. The domain $\Omega$ is 2-parabolic if and only if there exists a sequence of functions $\rho_{j} \in \operatorname{Lip}_{c}(\Omega)$ such that $0 \leq \rho_{j} \leq 1, \rho_{j}$ converges to 1 uniformly on every compact subset of $\Omega$ and

$$
\int_{\Omega}\left|\nabla \rho_{j}\right|^{2} d \mu_{g} \rightarrow 0
$$

Proof. First suppose $\Omega$ is 2-parabolic. Then every compact set $D \subset \Omega$, with nonempty interior satisfies $\operatorname{cap}(D, \Omega)=0$. We choose an exhaustion $D \subset D_{1} \subset D_{2} \subset \ldots \subset \Omega$ of $\Omega$ by compact subsets such that $\operatorname{cap}\left(D_{j}, \Omega\right)=0$ for all $j$. Hence we can find the function $\rho_{j} \in \operatorname{Lip}_{c}(\Omega)$ (using the fact that $\operatorname{Lip}_{c}(\Omega)$ is dense in $W_{0}^{1,2}(\Omega)$ ) such that $\rho_{j} \equiv 1$ on $D_{j}$ and $\int_{\Omega}\left|\nabla \rho_{j}\right|^{2} d \mu_{g} \leq 1 / j^{2}$. We have constructed the desired sequence $\rho_{j}$. Conversely, suppose there exists, a sequence $\rho_{j} \in \operatorname{Lip}_{c}(\Omega)$ with the stated properties. Then we can find a compact subset $B \subset \Omega$ and $j_{0}$ such that $\rho_{j} \geq 1 / 2$ for every $j \geq j_{0}$. It follows that $\operatorname{cap}(B, \Omega)=0$ 
The following lemma shows that the 2-parabolicity remains after removing the singular set of a Riemannian polyhedron.

Lemma 5.2. If $X$ is a 2-parabolic admissible Riemannian polyhedron and $E \subset X$ is a polar set, then $\Omega:=X \backslash E$ is 2-parabolic.

Proof. $X$ is 2-parabolic, so by Lemma 5.1, there are an exhaustion of $X$ and a sequence of function $\rho_{j} \in \operatorname{Lip}_{c}(X)$ such that $0 \leq \rho_{j} \leq 1$ and $\rho_{j} \rightarrow 1$ uniformly on each compact set, and $\int_{X}\left|\nabla \rho_{j}\right|^{2} d \mu_{g} \rightarrow 0$. Also by Lemma 2.11, there exist another sequence of functions $\varphi_{j}$ with support in $X \backslash E$ such that $\varphi_{j} \rightarrow 1$ on each compact set of $X \backslash E$ and $\int_{X}\left|\nabla \varphi_{j}\right|^{2} d \mu_{g} \rightarrow 0$. The functions $\rho_{j} \varphi_{j}$ on $\Omega$ provides the condition for 2-parabolicity in Lemma 5.1.

The following result is an extension of Theorem 5.2 in [27] to admissible Riemannian polyhedra.

Proposition 5.3. Let $\left(X, g, \mu_{g}\right)$ be 2-parabolic admissible Riemannian polyhedron. Suppose $f$ in $W_{\mathrm{loc}}^{1,2}(X)$ is a positive, continuous superharmonic function on $X$. Then $f$ is constant.

Proof. Since $f$ is continuous, for any $\epsilon$ and at any point $x_{0}$ in $X$ there exist a relatively compact neighborhood $B_{0}$ of $x_{0}$ such that $f(x)>f\left(x_{0}\right)-\epsilon$ on $\overline{B_{0}}$. $X$ is 2-parabolic, so cap $\left(B_{0}, X\right)=0$. Consider an exhaustion of $X$ by regular domains $U_{i}$ such that $B_{0} \Subset U_{1} \Subset U_{2} \Subset \ldots \Subset X$. By Corollary 11.25 in [6], such exhaustion exists.

There exist functions $u_{i}$ which are harmonic on $U_{i} \backslash \overline{B_{0}}, u_{i} \equiv 1$ on $B_{0}$ and $u_{i} \equiv 0$ on $X \backslash U_{i}$ (See [18] and also Lemma 11.17 and 11.19 in [6]). The maximum principle (see Theorem 5.3 in [13] or Lemma 10.2 in [6] for the comparison principle) implies that

$$
\left\{\begin{array}{l}
0 \leq u_{i} \leq 1 \\
u_{i+1} \geq u_{i} \text { on } U_{i}
\end{array}\right.
$$

Define the function $h_{i}=\left(f\left(x_{0}\right)-\epsilon\right) u_{i}$, we have $\lim _{i \rightarrow \infty} h_{i}=f\left(x_{0}\right)-\epsilon$. On the other hand $f \geq h_{i}$ on the boundary of $U_{i} \backslash \overline{B_{0}}$. By the comparison principle $f \geq h_{i}$ in $U_{i} \backslash \overline{B_{0}}$, so $f \geq f\left(x_{0}\right)-\epsilon$ on $X$. Letting $\epsilon \rightarrow 0$, we obtain $f \geq f\left(x_{0}\right)$ on $X$. If $f$ is non-constant, there exist $x_{1} \in X$ with $f\left(x_{1}\right)>f\left(x_{0}\right)$. By the same argument we obtain $f>f\left(x_{1}\right)$. This is a contradiction and thus $f$ is constant.

We prove the analogue of Theorem 1.2, for 2-parabolic admissible Riemannian polyhedra.

Proposition 5.4. Let $\left(X, g, \mu_{g}\right)$ be a 2-parabolic pseudomanifold. Let $f$ in $W_{\text {loc }}^{1,2}(X)$ be a continuous, weakly subharmonic function, such that $\|\nabla f\|_{L^{1}}$ and $\|\nabla f\|_{L^{2}}$ are finite. Then $f$ is harmonic.

Proof. Since $X$ is 2-parabolic, by Lemma 5.1 for every compact set $D \subset X$, and an arbitrary exhaustion $D \subset$ $D_{1} \subset D_{2} \subset \ldots \subset X$ of $X$ by compact subsets, there exist a sequence of functions $\rho_{j} \in \operatorname{Lip}_{c}(X)$ such that $\rho_{j} \equiv 1$ on $D_{j}$ and $\int_{X}\left|\nabla \rho_{j}\right|^{2} d \mu_{g} \leq 1 / j^{2}$.

$$
0 \leq-\int_{X}\left\langle\nabla \rho_{j}, \nabla f\right\rangle d \mu_{g} \leq\left(\int_{X}\left|\nabla \rho_{j}\right|^{2} d \mu_{g}\right)^{\frac{1}{2}}\left(\int_{X}|\nabla f|^{2} d \mu_{g}\right)^{\frac{1}{2}} \leq \frac{1}{j}\|\nabla f\|_{L^{2}}^{2} .
$$

By Lemma 4.1, there is a locally finite Borel measure $\mathbf{m}_{f}$ such that

$$
0<\int_{D} \mathbf{m}_{f} \leq \int_{X} \rho_{j} \mathbf{m}_{f} \leq\left|\int_{X}\left\langle\nabla \rho_{j}, \nabla f\right\rangle d \mu_{g}\right| \leq \frac{1}{j}\|\nabla f\|_{L^{2}}^{2} .
$$

Now let $h$ be an arbitrary test function in $\operatorname{Lip}_{c}(X)$ where its support is in $D$. We have

$$
0 \leq \int_{D} h \mathbf{m}_{f} \leq\left(\sup _{X} h\right) \frac{\|\nabla f\|_{L^{2}}^{2}}{j}
$$

and so $f$ is harmonic on $X$. 
Similarly we have the following result generalizing Theorem 5.9 in [27].

Proposition 5.5. Let $\left(X, g, \mu_{g}\right)$ be 2-parabolic admissible Riemannian polyhedron. Let $f$ in $W_{\mathrm{loc}}^{1,2}(X)$ be a harmonic function such that $\|\nabla f\|_{L^{2}}$ is finite. Then $f$ is constant.

Proof. Set

$$
f_{i}=\max (-i, \min (i, f)) .
$$

Let $U_{j}$ be an exhaustion of $X$ by regular domains $U_{j} \subset U_{j+1} \Subset X$. There is a continuous function $u_{i, j}$ such that $u_{i, j}$ are harmonic on $U_{j}$ and $u_{i, j}=f_{i}$ in $X \backslash U_{i}$. Also $u_{i, j}$ is continuous on $X$ and $\left\|\nabla u_{i, j}\right\|_{L^{2}}$ is finite. We have $-i \leq u_{i, j} \leq i$. According to Theorem 6.2 in [13], $u_{i, j}$ are Hölder continuous (after correction on a null set), and since they are uniformly bounded, by Theorem 6.3 in [13], they are locally uniformly Hölder equicontinuous and by Theorem 9.37 in [6], there is a subsequence which converges locally uniformly to some $u_{i}$ as $j \rightarrow \infty$. Note that the definition of harmonicity as in [6] is consistent with our definition. The function $u_{i}$ is bounded and harmonic and hence is constant. Moreover $u_{i, j}-f_{i} \in L_{0}^{1,2}$ and so $f_{i} \in L_{0}^{1,2}$. Therefore

$$
\int_{X}|\nabla f|^{2} d \mu_{g}=\lim _{i \rightarrow \infty} \int_{X}\left\langle\nabla f, \nabla f_{i}\right\rangle d \mu_{g}=0
$$

and $f$ is constant.

By use of Lemma 4.6 and the above propositions, the proofs of Corollaries 1.6 and 1.7 are straightforward.

Acknowledgement: The original version of this work was part of my Ph.D. dissertation. I thank my advisor Professor Marc Troyanov for his guidance and support. I also thank my Ph.D. exam jury members Professors Buser, Wenger and particularly Professor Naber who suggested an additional bound on $|\nabla f|$ as hypothesis in Theorem 1.2, and so automatically on $e(u)$ in Theorems 1.3 and 1.4. I am very grateful to thank Professor Goufang Wei who I met as a postdoc in MSRI, for suggesting that I read papers by $[28,30,33]$ which allowed me to remove this assumption in Theorems 1.2, 1.4.

\section{References}

[1] Luigi Ambrosio, Nicola Gigli, and Giuseppe Savaré. Metric measure spaces with Riemannian Ricci curvature bounded from below. Duke Math. J., 163(7):1405-1490, 2014.

[2] L. Ambrosio, N. Gigli, and G. Savaré. Calculus and heat flow in metric measure spaces and applications to spaces with Ricci bounds from below. ArXiv e-prints, June 2011.

[3] L. Ambrosio, A. Mondino, and G. Savaré. On the Bakry- \'Emery condition, the gradient estimates and the Local-toGlobal property of $\operatorname{RCD}^{\star}(K, N)$ metric measure spaces. ArXiv e-prints, September 2013.

[4] Luigi Ambrosio and Paolo Tilli. Topics on analysis in metric spaces, volume 25 of Oxford Lecture Series in Mathematics and its Applications. Oxford University Press, Oxford, 2004.

[5] Kathrin Bacher and Karl-Theodor Sturm. Localization and tensorization properties of the curvature-dimension condition for metric measure spaces. J. Funct. Anal., 259(1):28-56, 2010.

[6] Anders Björn and Jana Björn. Nonlinear potential theory on metric spaces, volume 17 of EMS Tracts in Mathematics. European Mathematical Society (EMS), Zürich, 2011.

[7] Dmitri Burago, Yuri Burago, and Sergei Ivanov. A course in metric geometry, volume 33 of Graduate Studies in Mathematics. American Mathematical Society, Providence, RI, 2001.

[8] Jeff Cheeger. On the Hodge theory of Riemannian pseudomanifolds. In Geometry of the Laplace operator (Proc. Sympos. Pure Math., Univ. Hawaii, Honolulu, Hawaii, 1979), Proc. Sympos. Pure Math., XXXVI, pages 91-146. Amer. Math. Soc., Providence, R.I., 1980.

[9] Jingyi Chen. On energy minimizing mappings between and into singular spaces. Duke Math. J., 79(1):77-99, 1995.

[10] J. Cheeger. Differentiability of Lipschitz functions on metric measure spaces. Geom. Funct. Anal., 9(3):428-517, 1999.

[11] Georgios Daskalopoulos and Chikako Mese. Harmonic maps from a simplicial complex and geometric rigidity. J. Differential Geom., 78(2):269-293, 2008.

[12] Georgios Daskalopoulos and Chikako Mese. Harmonic maps between singular spaces I. Comm. Anal. Geom., 18(2):257-337, 2010. 
[13] J. Eells and B. Fuglede. Harmonic maps between Riemannian polyhedra, volume 142 of Cambridge Tracts in Mathematics. Cambridge University Press, Cambridge, 2001.

[14] M. Erbar, K. Kuwada, and K.-T. Sturm. On the Equivalence of the Entropic Curvature-Dimension Condition and Bochner's Inequality on Metric Measure Spaces. ArXiv e-prints, March 2013.

[15] N. Gigli. On the differential structure of metric measure spaces and applications. ArXiv e-prints, May 2012.

[16] Nicola Gigli, Kazumasa Kuwada, and Shin-Ichi Ohta. Heat flow on Alexandrov spaces. Comm. Pure Appl. Math., 66(3):307-331, 2013.

[17] Mikhail Gromov and Richard Schoen. Harmonic maps into singular spaces and $p$-adic superrigidity for lattices in groups of rank one. Inst. Hautes Études Sci. Publ. Math., (76):165-246, 1992.

[18] Vladimir Gol'dshtein and Marc Troyanov. Axiomatic theory of Sobolev spaces. Expo. Math., 19(4):289-336, 2001.

[19] Vladimir Gol'dshtein and Marc Troyanov. Capacities in metric spaces. Integral Equations Operator Theory, 44(2):212242, 2002.

[20] R. E. Greene and H. Wu. Smooth approximations of convex, subharmonic, and plurisubharmonic functions. Ann. Sci. École Norm. Sup. (4), 12(1):47-84, 1979.

[21] Piotr Hajłasz. Sobolev spaces on an arbitrary metric space. Potential Anal., 5(4):403-415, 1996.

[22] Piotr Hajłasz and Pekka Koskela. Sobolev met Poincaré. Mem. Amer. Math. Soc., 145(688):x+101, 2000.

[23] Juha Heinonen and Pekka Koskela. Quasiconformal maps in metric spaces with controlled geometry. Acta Math., 181(1):1-61, 1998.

[24] Stefan Hildebrandt. Liouville theorems for harmonic mappings, and an approach to Bernstein theorems. In Seminar on Differential Geometry, volume 102 of Ann. of Math. Stud., pages 107-131. Princeton Univ. Press, Princeton, N.J., 1982.

[25] Stefan Hildebrandt. Harmonic mappings of Riemannian manifolds. In Harmonic mappings and minimal immersions (Montecatini, 1984), volume 1161 of Lecture Notes in Math., pages 1-117. Springer, Berlin, 1985.

[26] S. Hildebrandt, J. Jost, and K.-O. Widman. Harmonic mappings and minimal submanifolds. Invent. Math., 62(2):269$298,1980 / 81$.

[27] Ilkka Holopainen. Nonlinear potential theory and quasiregular mappings on Riemannian manifolds. Ann. Acad. Sci. Fenn. Ser. A I Math. Dissertationes, (74):45, 1990.

[28] B. Hua, M. Kell, and C. Xia. Harmonic functions on metric measure spaces. ArXiv e-prints, August 2013.

[29] Renjin Jiang. Lipschitz continuity of solutions of Poisson equations in metric measure spaces. Potential Anal., 37(3):281-301, 2012.

[30] Renjin Jiang. Cheeger-harmonic functions in metric measure spaces revisited. J. Funct. Anal., 266(3):1373-1394, 2014.

[31] Jürgen Jost. Equilibrium maps between metric spaces. Calc. Var. Partial Differential Equations 2(2):173-204, 1994.

[32] Jürgen Jost. Nonlinear Dirichlet forms. New directions in Dirichlet forms, 1-47, AMS/IP Stud. Adv. Math., 8, Amer. Math. Soc., Providence, RI, 1998.

[33] M. Kell. A Note on Lipschitz Continuity of Solutions of Poisson Equations in Metric Measure Spaces. ArXiv e-prints, July 2013.

[34] Nicholas J. Korevaar and Richard M. Schoen. Sobolev spaces and harmonic maps for metric space targets. Comm. Anal. Geom., 1(3-4):561-659, 1993.

[35] Nicholas J. Korevaar and Richard M. Schoen. Global existence theorems for harmonic maps to non-locally compact spaces. Comm. Anal. Geom. 5 (1997), 5(2):333-387, 1997.

[36] Pekka Koskela, Kai Rajala, and Nageswari Shanmugalingam. Lipschitz continuity of Cheeger-harmonic functions in metric measure spaces. J. Funct. Anal., 202(1):147-173, 2003.

[37] Kazuhiro Kuwae and Takashi Shioya. On generalized measure contraction property and energy functionals over Lipschitz maps. Potential Anal., 15(1-2):105-121, 2001. ICPA98 (Hammamet).

[38] Kazuhiro Kuwae and Takashi Shioya. Sobolev and Dirichlet spaces over maps between metric spaces. J. Reine Angew. Math., 555:39-75, 2003.

[39] Kazuhiro Kuwae and Karl-Theodor Sturm. On a Liouville type theorem for harmonic maps to convex spaces via Markov chains. In Proceedings of RIMS Workshop on Stochastic Analysis and Applications, RIMS Kôkyûroku Bessatsu, B6, pages 177-191. Res. Inst. Math. Sci. (RIMS), Kyoto, 2008.

[40] John Lott and Cédric Villani. Weak curvature conditions and functional inequalities. J. Funct. Anal., 245(1):311-333, 2007.

[41] John Lott and Cédric Villani. Ricci curvature for metric-measure spaces via optimal transport. Ann. of Math. (2), 169(3):903-991, 2009.

[42] Shin-ichi Ohta. On the measure contraction property of metric measure spaces. Comment. Math. Helv., 82(4):805-828, 2007.

[43] Athanase Papadopoulos. Metric spaces, convexity and nonpositive curvature, volume 6 of IRMA Lectures in Mathematics and Theoretical Physics. European Mathematical Society (EMS), Zürich, 2005.

[44] Nageswari Shanmugalingam. Newtonian spaces: an extension of Sobolev spaces to metric measure spaces. Rev. Mat. Iberoamericana, 16(2):243-279, 2000.

[45] Richard Schoen and Shing Tung Yau. Harmonic maps and the topology of stable hypersurfaces and manifolds with non-negative Ricci curvature. Comment. Math. Helv., 51(3):333-341, 1976. 
[46] Karl-Theodor Sturm. On the geometry of metric measure spaces. II. Acta Math., 196(1):133-177, 2006.

[47] M. Troyanov. Parabolicity of manifolds. Siberian Adv. Math., 9(4):125-150, 1999.

[48] Yuanlong Xin. Geometry of harmonic maps. Progress in Nonlinear Differential Equations and their Applications, 23. Birkhäuser Boston Inc., Boston, MA, 1996.

[49] Shing Tung Yau. Some function-theoretic properties of complete Riemannian manifold and their applications to geometry. Indiana Univ. Math. J., 25(7):659-670, 1976.

[50] Hui-Chun Zhang and Xi-Ping Zhu. Yau's gradient estimates on Alexandrov spaces. J. Differential Geom., 91(3):445-522, 2012. 